\title{
Die Erscheinungen der Wasserbewegung und ihre Wirkung auf Sedentarier im mediterranen Felslitoral
}

\author{
RUPERT RIEDL \\ I. Zoologisches Institut der Universität Wien, Österreich
}

\begin{abstract}
The phenomena of water movement and their effect on sedentary organisms in the mediterranean rocky littoral. Water movement is shown to be a very important environmental factor. It largely determines the distribution of substrates and the position and shape of organisms, especially in sedentary inhabitants of rocky littoral areas. In spite of its great ecological significance, water movement has not yet been measured in these areas. However, earlier information obtained in the fields of oceanography, geology and geomorphology, as well as in connection with marine construction, offer sufficient background data to assess the major hydrodynamic phenomena in littoral habitats. Thus scientific diving made it possible in numerous cases to relate cause and effect. The relations observed are documented by one example in each case and analysed on the basis of 5 different aspects.
\end{abstract}

\section{EINLEITUNG}

Längst hat man die Wasserbewegung als einen Faktor erkannt, der die Anordnung der marinen Lebensräume und der Organismen ebenso stark beeinflußt wie vielfach auch deren Entwicklung und Fortkommen. In der Litoralforschung ist man von der erstrangigen Bedeutung dieses Faktors so überzeugt, daß man vielfach unter Exposition ausschließlich die Bewegungsexposition eines Standortes meint. Besonders die in Fels litoral geübte Methode der Taucherei hat eine ganze Reihe durch direkte Anschau ung gewonnene Einsichten in das Phänomen geliefert (Abel 1959, RIEDL 1959, $1963,1964 a$ ).

Angesichts dieser Tatsache muß es auffallen, daß die Wasserbewegung in der unmittelbaren Nähe der Standorte des Felslitorales (anders liegen die Verhältnisse an Sediment-Küsten) noch nicht vermessen worden ist. Das kommt in erster Linie daher, daß schon beim Herantreten an diese Aufgabe klar wird, daß wir noch gar nicht in der Lage sind, die Fragestellung treffend zu formulieren, denn die Wirkungsweise der Wasserbewegung ist ganz offensichtlich mehrgliedrig, und zudem ist noch nicht ausgemacht, wie die vielfältigen, als expositionsabhängig anzunehmenden Gestalts- oder Verteilungs-Erscheinungen mit den einzelnen Aspekten der Wasserbewegung in Beziehung stehen. Wir wissen darum in vielen Fällen nicht einmal, welcher der physikalischen Ausdrücke zu vermessen wäre, der Staudruck, die Durchflußmenge, die Teilchengeschwindigkeit, der Rhythmus oder die Wasserversetzung, die jeweiligen Maxima, die Minima oder die Mittelwerte. Und selbst wenn angenommen werden 
könnte, daß in einem Fall das Maximum der Teilchengeschwindigkeit von Einfluß sein muß, vermöchten wir noch immer nicht anzugeben, welche Zeitintervalle zur Kennzeichnung signifikant sein dürften, die stündlichen, täglichen oder jährlichen Maxima (RredL 1959, 1963). Dieser Gegensatz ist in einem ersten Schritt dadurch überbrüdkbar, daß aus dem Erfahrungsschatz dreier benachbarter Disziplinen die einschlägigen Phänomene ausgewählt und interpolierend den Verhältnissen im Felslitoral angelegt werden. 1. Die Ozeanographie böte hinsichtlich der Ursachen und der Erscheinungen der Wasserbewegung ein für unsere Zwecke längst zureichendes Erfahrungsvolumen (Defant 1929, Thorade 1931, Sverdrup et al. 1954, Dietrich \& Kalle 1957). Aber sie befaßt sich vornehmlich mit den Bewegungserscheinungen der offenen Wassermassen. Die minitiösen Umformungen der Bewegung innerhalb kleinräumiger Bodenkonfigurationen und Organismenbestände sind für sie naturgemäß von ganz untergeordnetem Interesse.

2. Die maritime Geologie und Geomorphologie (Ubersichten bei Cviırc 1924, Shepard 1948, Kuenen 1950, Guilcher 1954) bertihren in vieler Hinsicht Fragen der Wasserbewegung im unmittelbaren Küstengebiet. Aber es sind überwiegend Abrasions- und Errosionsfragen sowie solche des Sedimenttransportes, die sie interessieren müssen.

3. Der maritime Wasserbau endlich stützt sich bereits auf eine Fülle metrisch gewonnener Erfahrungen, die, wenn auch in der Hauptsache die Festigkeit von Kunstbauten ins Auge gefaßt sind (etwa: Volrbrecht 1954, Domzig 1955, IrBARREN 1953), unserer Fragestellung besonders greifbare Ansatzpunkte liefern.

Ohne noch über Messungen im Felslitoral zu verfügen, ist es nun schon möglich, eine Fülle biologischer Erscheinungen im Zusammenhang mit jenen der Wasserbewegung $z u$ interpretieren. In der vorliegenden Schrift soll nun versucht werden, ein möglichst geschlossenes Bild der bislang erkannten Beziehung zu liefern, indem auch jede derselben durch ein Beispiel beleuchtet wird. Eine solche Vorausordnung der Beziehungen, wie sie hier noch vor Erreichen des metrischen Forschungsabschnittes und zu dessen Anregung angestrebt wird, verlangt, 5 verschiedene Perspektiven anzuwenden. Zuerst seien die biologischen Beispiele für die Abhängigkeit von den Grunderscheinungen und Bewegungsweisen vorgelegt, dann diejenigen für die Bewegungsgefälle angeschlossen, schließlich solche der einfachen und komplexen Wirkungen systematisch dargestellt.

\section{DIE GRUNDERSCHEINUNGEN}

Unter den Erscheinungen eines bewegten Wasserkörpers seien hier die unmittelbaren physikalischen Ausdrücke desselben, oft ein und desselben Vorganges, verstanden. Wiewohl diese verflochten, sich vielfach gegenseitig bestimmend, zusammenhängen, bietet doch jeder von ihnen in biologischer Sicht einen scheinbar eigenen Wirkungskreis, der vorerst einmal abzugrenzen wäre.

Die Sta ud rucke bestimmen in erster Linie die Festigkeit des Substrates, weniger die mechanische Resistenz der Individuen, denn die starren Bautypen bieten meist kleine Querschnitte (geringe Angriffsflächen), die geschmeidigen Formen leiten die Bewegung $a b$, und für die Krustenformen spielen sie kaum eine Rolle. Die ungeheuren 
Kräfte, die an einer Mole oder an einer Felswand bei schwerer See auftreten können (bis $100 \mathrm{t} / \mathrm{m}^{2}$ wurden vermessen), scheinen für die Organismenwelt nahezu geringfügig. Ein Seegang, der beispielsweise einen Felsblock mit einem Gewidht von 15 Tonnen zu versetzen vermag, löst nicht ein gesundes Individuum der Seepocke Balarus perforatus von der Unterlage. Und die mit dem Auf- und Ablaufen der Wogen rasch wechselnden hydrostatischen Drucke spielen offenbar überhaupt keine Rolle.

Die Durchflu Bmenge des Wassers durch einen bestimmten Querschnitt ist in erster Linie für die Ernährung und Belïftung wichtig. Wenn man bedenkt, daß ein Sedentarier-Bestand mit der Fläche von $1 \mathrm{~m}^{2}$ pro Stunde ein Wasservolumen von 1,5 Tonnen, unter Umständen von 7 Tonnen za filtrieren vermag, wird die Bedeutung

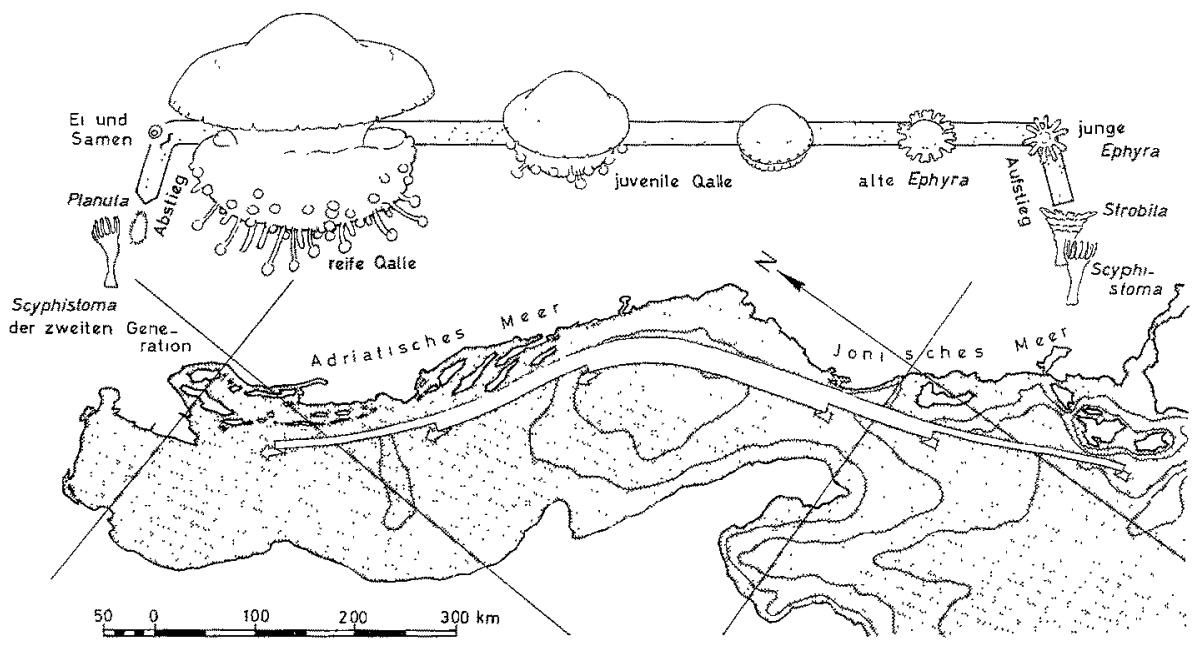

Abb. 1: Die Wirkung der Wasserversetzung auf den Transport pelagischer Generationen (am Beispiel einer Generation von Cotylorbiza tuberculata im Adriagebiet). Die weiße Pfeilgruppe gibt die Strecke an, über welche eine Generation im Laufe von 4 Monaten bei einer mittleren Wasserversetzung von 9,5 oder $2,5 \mathrm{~cm} / \mathrm{sek}(=90 \mathrm{~m} / \mathrm{h},=2,2 \mathrm{~km} / \mathrm{Tag})$ in Richtung der örtlichen Oberflächenströmung verschoben wird. Entlang des punktierten Pfeiles sind die Stadien eingetragen. Die Winkel der Pfeile deuten das Aufsteigen der Ephyren und das Absteigen der Planulen an

des Wasserwechsels sofort offensichtlich. Dabei schließt diese Kalutation (RIEDL 1964b) nur die Leistung der wichtigsten "aktiven Filtrierer" der Sedentarier ein, Porifera, einige Polychaeta-Sedentaria, Lamellibranchiata, Ascidiacea und z. T. die Cirripedia. Die „passiven Filtrierer" der Sedentarier, die Mehrzahl der benthischen Cnidaria, werden überhaupt nur durch vorbeistreichendes Wasser begünstigt, und die Leistung der wahrscheinlich zahlreichen Typen aktiver und passiver Filtrierer aus Errantier-Gruppen beginnen wir eben erst kennenzulernen (Gastropoda: WERNER 1951, 1953, Ophiuroidea: RIEDL 1956, 1963, MAGNUS 1963a, Crinoidea: MaGNus 1963b).

Die Wasserversetzung wiederum, also die Transportstrecke, die ein Wasserkörper innerhalb bestimmter Zeit (oder bestimmter Abschnitte eines rhythmischen Ablaufes) erfährt, nimmt größten Einfluß auf die Verdriftung aller ins Medium freigegebener Fortpflanzungsprodukte und Generationen. Sie wirkt maßgeblich auf Be- 
fruchtung und Verbreitung der Mehrzahl der Sedentarier ein. So ist z. B. nach den Strömungsverhältnissen und der pelagischen Lebenszeit zu errechnen, daß die im späten Frühling allmählich mit geschlechtsreif werdenden Individuen in Dalmatien ankommenden großen Schwärme der Wurzelqualle Cotylorbiza tuberculata (Abb. 1) während des vorausgegangenen Winters in albanischen Gewässern, in Corfu oder sogar in der Kreta-Straße als Ephyren von den dort ansässigen Scyphistoma-Polypen freigeworden sind, je nachdem man eine mittlere Wasserversetzung von 2,5, 5 oder $10 \mathrm{~cm} / \mathrm{sek}$ anzunehmen hat.

Die Teilchengeschwindigkeit ist von fundamentaler Bedeutung. Erstens kann man aus ihr Durchflußmengen und eventuell entstehende Staudrucke errechnen und damit auf die Bedingungen von Substratfestigkeit, Resistenz der Organismen, Ernährung und Belüftung schließen. Zweitens wird die Sedimentverteilung und damit die Verteilung der Bodentypen von ihr festgelegt, und drittens bestimmen die ihr eigenen Transportbedingungen Säuberung, Bescheuern und Verschütten der sedentären Organismen. Der Umfang des Wasserwechsels und der Wasserversetzung läßt sich aus der Geschwindigkeit ebenso ermitteln, sofern der Rhythmus der Bewegung bekannt ist. Unter dieser Voraussetzung kann die Teilchengeschwindigkeit auch als ein allgemeines Hilfsmaß verwendet werden.

Der Rhythmus der Bewegung, ob es sich um gerade oder pendelnde, lang- oder kurzschwingende, periodische oder unregelmäßige Bewegungen handelt, bestimmt und verändert die Wirkung aller Grunderscheinungen der Wasserbewegung. Man kann darum von verschiedenen Bewegungsweisen sprechen. Der folgende Absatz soll sich mit diesen befassen.

\section{DIE BEWEGUNGSWEISEN}

Innerhalb der ozeanisch gesehenen Bewegungserscheinungen nehmen, in den vorliegenden Belangen, die Bewegungen der Medium- und Dichtegrenzen eine Sonderstellung ein.

Hierher zählen (1.) Windstau, Gezeit und Seiches, Windsee, Dünung und Brandung, sofern sie als lang- und kurzzeitige Wasserstandsschwankungen betrachtet werden. Unter diesem Gesichtspunkt beeinflussen die genannten Bewegungsweisen lediglich Rhythmus und Ausmaß des Trockenfallens im Gezeitengebiet und in den unmittelbar anschließenden Küstenabschnitten. Hierher zählen (2.) auch die Bewegungen an den inneren Dichtegrenzen, interne Gezeiten, -Brandungen, -Trägheits- und -Stabilitätsschwingungen. Als Pegeländerung allein genommen wirken sie auf die Bodenorganismen in der jeweils kritischen Tiefe nur insofern ein, als sie den Rhythmus und das Ausmaß bestimmen, mit welchem jene mit unterschiedlichen Wasserkörpern in Berürung kommen.

Beide Gruppen von Bewegungsweisen betreffen unseren Problemkreis nur am Rande, gewissermaßen als Begleiterscheinungen der inneren Bewegungsweisen selbst. Die Bewegung der Mediumgrenze ist als bestimmender Faktor gut sichtbar und als solcher vielfach studiert worden. Sie findet ihren biologischen Ausdruck in den Zonationen des Eu- und Supralitorales. Die Bewegung der inneren Dichtegrenzen wurde 
ungleich später erschlossen. Über ihre Wirkung auf Benthos-Organismen scheint noch nichts bekannt zu sein.

Hinsichtlich der Bew egungs weise des Mediums selbst (nicht seiner Oberflächen) lassen sich nach biologischen Gesichtspunkten vorerst drei Gruppen bilden: streichende, schwingende und zerreißende Wasserkörper. Man könnte fast Strömung, Orbitalbewegung und Brandung sagen, doch die biologischen Begriffe decken sich mit den bewährten ozeanographischen Einheiten - wie zu zeigen sein wird - nur teilweise; darum ist es an dieser Stelle, wo es auf die Gegenüberstellung ankommt, nötig, sie anders zu benennen.

\section{Der streichende Wasserkörper}

In diese biologische Kategorie von Bewegungsweisen gehören neben den Strömungen im engeren Sinne auch jene Erscheinungen der Wasserversetzung, welche gemeinsam mit den langen Schwingungen auftreten; die Strömungen der Gezeiten, Re-sonanzschwingungen und Seiches. Hinsichtlich der Erscheinungen der Durchflußmenge und der Teilchengeschwindigkeit scheint es für die Sedentarier des Felslitorales gleichgültig zu sein, ob das Medium nur in einer Richtung oder aber auch in der Gegenrichtung an ihnen vorbeigeführt wird. Jedenfalls ist nach der Besiedlungsweise einmal an den überwiegend gerichteten Strömungen ausgesetzten offenen Küstenstreifen, ein andermal an den überwiegend gezeitenbeströmten Hälsen tiefer Buchten (wie sie in Dalmatien vielfach gut ausgebildet sind) kein Unterschied nachzuweisen.

Diese streichende Wasserbewegung herrscht unterhalb des pendelnden Wasserkörpers im ganzen Meere vor. Dabei ist die gerade, eindimensionale, der Küste parallele Form des Streichens nur in unmittelbarer Küstennähe zu erwarten und ledig." lich in den steilen Teilen des Küstendecliviums (in dem sich - im Sinne von Lorenz 1863 - fast immer Felslitoral vorfindet) mit Gewißheit vorauszusetzen (Abb. 2). Uber den ebenen oder nur wenig geneigten Böden, besonders unterhalb des Decliviums, sind ganz überwiegend girlandenförmig oder kreisend fortschreitende, also zw eidimensionale, Strombahnen zu erwarten, entsprechend der Bewegungssumme aus permanenten Strömungen und der Wasserversetzung durch die langen Schwingungen. Es resultieren kompliziert ellipsen- und schlingenförmige Bahnen der Wasserteilchen, welche durch die schwingungsunabhängigen Strömungen und nach dem Verhältnis der Geschwindigkeiten schrittweise verschoben werden. Folglich wird ein Sedentarier im Declivium nur von zwei einander gegenüberliegenden Seiten beströmt, ein solcher der Bodenebenen jedoch aus allen horizontalen Richtungen.

Der optimale Fangapparat der "passiven Filtrierer“ und Sammler muß darum im linearen Strömungsgebiet die querstehende Fläche, im flächigen der Radiärbau sein, wobei die Hauptachse nun normal zur Strömung steht. Ganz entsprechend überwiegen innerhalb des Delicliviums flache Wuchsformen wie Halocordyle disticha, Eudendrium rameum, Plumularia setacea, Aglaopbenia pluma, Eunicella cavolinii, auf den Ebenen dagegen die radiären Typen, etwa Eudendrium ramosum, Nemertesia antennina, Alcyonium palmatum, Lytocarpia myriopbyllum, Isidella elongata (Abb. 2). Dabei ist es von Interesse festzustellen, auf welche Weisen der streng flächige Wuchs des Typus der Plumulariidae aufgelöst $\mathrm{zu}$ werden vermag oder umgekehrt die radiäre Anlage 


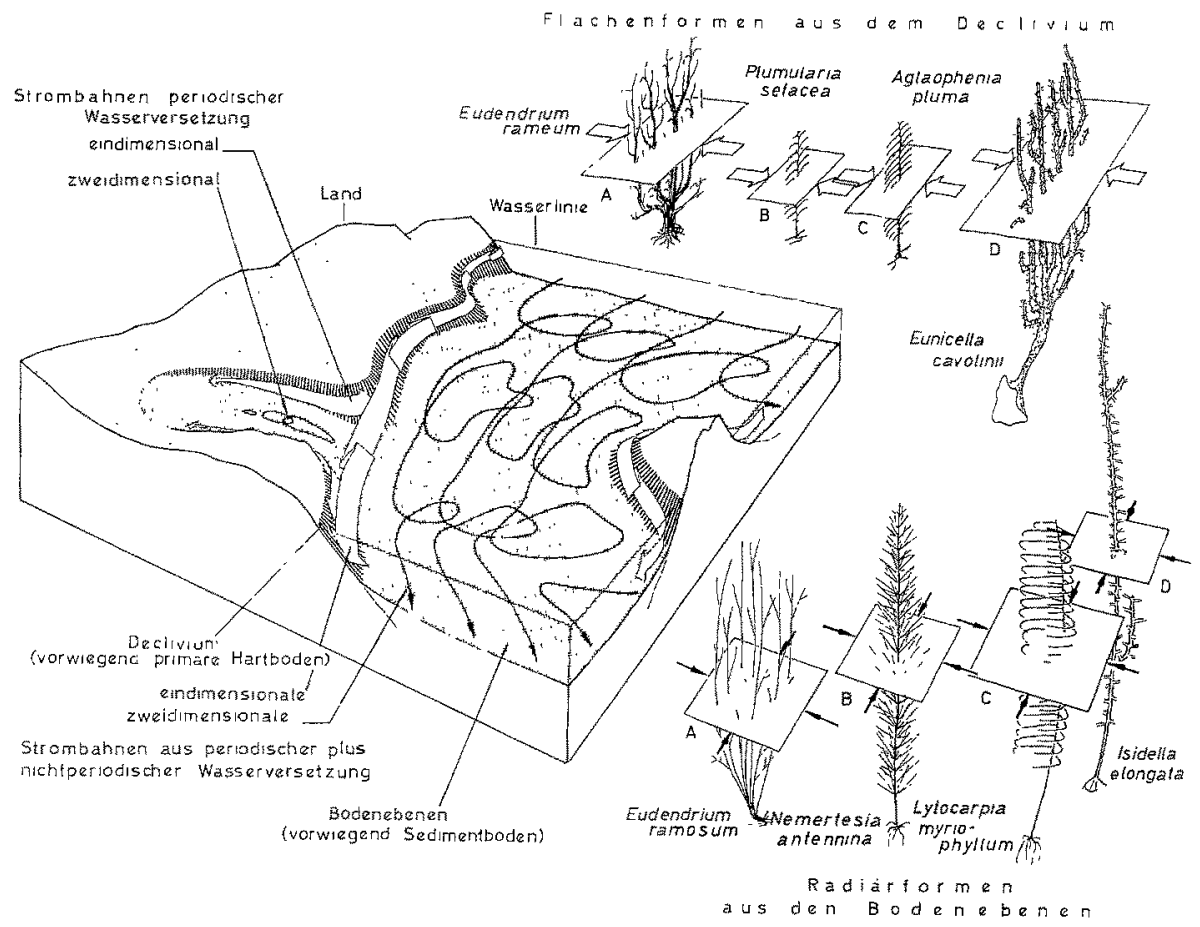

Abb. 2: Die Wirkung des ein- und zweidimensional streichenden Wasserkörpers auf die Verteilung der flächigen und radiären Typen passiver Filtrierer. Das Blockdiagramm gibt überhöht einen Küstenausschnitt von rund vier Quadratmeilen mit Tiefen bis etwa $50 \mathrm{~m}$ wieder. Die Falten der eindimensionalen Strombahnen (periodisch plus nichtperiodische Wasserversetzung) geben das Rücklaufen der periodischen Komponente an (den Zeitpunkt des „Kenterns" der zusammengesetzten Strömung im Declivium). Als Wuchsformen-Beispiele sind jeweils nahe verwandte Arten verwendet: A Gattung Eudendrium, B Unterfamilie Plumulariinae, C Aglaopheniinae, D Ordnung Gorgonaria. (Die eingesetzten Flächen veranschaulichen die Querschnitte der Formen; die entsprechenden Strombahnen sind hinzugefügt.)

der polysiphonen Eudendriidae und der Gorgonaria zum reinen Flächenbau geleitet wird (s. S. 179 bis 181).

Die Geschwindigkeit der streichenden Bewegung hält sich in der Regel zwischen 1 und $40 \mathrm{~cm} / \mathrm{sek}$, die Staudrucke bleiben unter der Schwelle biologischer Wirksamkeit, und die $\mathrm{Zu}$ - und Abfuhr des Wasser erfolgt über zureichend große Entfernungen. Auch die Richtungen sind im üblicherweise steilen Felslitoral gleich, nämlich stets parallel zur Küste. Die senkrecht zur Küste laufende Wasserversetzung in Folge der Pegelschwankung ist in unmittelbarer Nähe schroffer Küsten praktisch zu vernachlässigen. So kommt es, daß Strömungen und lange Schwingungen biologisch als ein einheitlicher Strömungsaspekt gemeinsam wirken, sich summierend und reduzierend entlang der Mehrzahl der Felsküsten den rhythmisch erfolgenden Vorbeischub des Wassers bewerkstelligen.

Hinsichtlich der Erscheinung der Wasserversetzung wirken Strömungen und lange Schwingungen zum Teil verschieden, nachdem die letzteren das versetzte Wasser nahe 


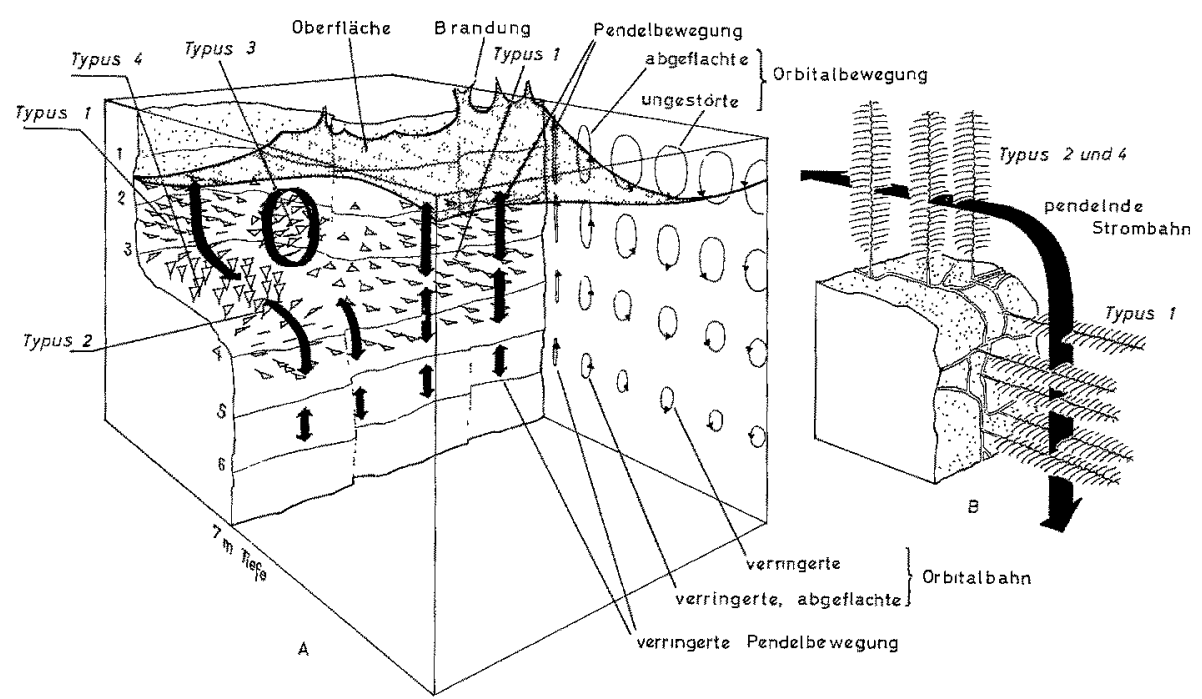

Abb. 3: Die Wirkung des pendelnden Wasserkörpers auf die Orientierung der Flächentypen passiver Filtrierer; in Abhängigkeit von der Substratgestalt. $A$ Überhöhtes Blockdiagramm eines Küstenausschnittes mit rund $25 \mathrm{~m}$ Länge und $7 \mathrm{~m}$ Tiefe. $B$ Ausschnitt. Die gestielten Dreiecke in $A$ sowie die Fiedern in $B$ stehen für primär flächige Hydroiden (Halocordyle disticha, Kirchenpaueria pinnata, Plamularia setacea sowie die Aglaophenia-Arten septifera, tubulifera und die var. octodonta der pluma). Orientierung nach Typ - 1 horizontal, 2 vertikal-küstenparallel, 3 verwirbelt und 4 vertikal-küstennormal

an seinen Ursprungsort zurückbringen, erstere aber nicht. Für die Verbreitung der Fortpflanzungszellen und der kurzlebigen Larven mag der Unterschied gering sein. Für die Verbreitungsbedingungen, welche planktische Generationen und langlebige Larven finden, müssen dagegen ungleiche Wirkungen angenommen werden. Die Stärke des Streichens hängt sehr von den örtlichen Bedingungen $a b$. Namentlich in Kanälen und Buchteingängen geringer Querschnitte nehmen die Werte zu. Die geringfügige $\mathrm{Ab}$ nahme gegen die Tiefe, so sich eine solche überhaupt generell postulieren läßr, kann aber in dem engen Bereich, den das Felslitoral bestreicht, vorerst vernachlässigt werden.

\section{Der schwingende Wasserkörper}

Diesen kennzeichnen jene kurzen Schwingungen, die an der Oberfläche als Windsee oder Dünung in Erscheinung treten und die Wasserteilchen in senkrecht kreisenden, orbitalen Bahnen bewegen; für das Benthos unter der Voraussetzung, daß die Bewegung wohl bis zum Grunde reicht, der Wasserkörper aber noch nicht - wie bei zu geringer Tiefe - zerreißt. Ozeanographisch gesehen handelt es sich um jenen Ausschnitt der inneren Bewegungserscheinung des Seeganges, der bereits die Brandung vorbereitet.

Die senkrecht laufenden Orbitalbahnen bleiben dabei annähernd am gleichen Ort und stehen in der Regel, der Fortschreiterichtung des Seeganges entsprechend, senkrecht auf die Küste. Der Durchmesser der Bahnen entspricht an der Oberfläche der 
Wellenhöhe, die Umlaufzeit dem Wellendurchgang. Die Geschwindigkeit der Teilchen ergibt sich damit aus Wellenhöhe und Wellengeschwindigkeit und liegt ïblicherweise bei Wellenhöhen zwischen $1 / 4$ bis $5 \mathrm{~m}$ zwischen 40 und $200 \mathrm{~cm} / \mathrm{sek}$. Entsprechend übertrifft sie die Geschwindigkeit der Strömungen in Oberflächennähe praktisch immer, gewöhnlich um eine, unter Umständen um zwei Dezimalen. Gegen die Tiefe nimmt der Durchmesser der Orbitalbahnen und damit die Geschwindigkeit der Teilchen ab. Diese Abnahme hängt besonders von der Wellenlänge ab. Die Orbitalbewegung verschwindet bei einer Tiefe, die annähernd der Wellenlänge entspricht. In unmittelbarer Bodennähe werden die Kreisbahnen zu immer flacheren Ellipsen und endlich zu geraden Pendelbewegungen parallel zum Bodenprofil (Abb. 3). Dabei bleiben dieselben (wie die Orbitalbahnen) senkrecht auf die Küste und normal zur Streichrichtung des Küstenwassers gerichtet. Mit abnehmender Wassertiefe wird der Pendelausschlag und damit die Teilchengeschwindigkeit größer, ohne daß sich aber etwas am harmonischen Ablauf der Bewegung änderte. Erst bei einer Tiefe, die nur mehr der $21 / 2$ fachen Wellenhöhe entspricht, ändert sich die Bewegungsweise wesentlich (s. Seite 167).

Die Anordnung jener passiven Filtrierer, deren Bauplan eine flächige Wuchsform nahelegt und von welchen bekannt ist (RIEDL 1959), daß sie ihre Fläche senkrecht in die Strömung stellen, läßt die herrschenden Verhältnisse vorerst sogar leichter abschätzen, als die schwierigen hydrodynamischen Beobachtungen und Berechnungen. Das kommt offenbar daher, daß die Organismen aus den verschiedenen Unregelmäßigkeiten der Bewegung bereits jenen signifikanten Mittelwert gezogen haben, welchen wir physikalisch erst aus einer sehr großen Zahl von Messungen ermitteln müßten. Die Arten Halocordyle disticha, Thecocaulus diaphanus, Plumularia setacea und die Aglaopbenia-Arten pluma f. octodonta, septifera und tubulifera lassen vier Pendelweisen unterscheiden (Abb. 3). Der häufggste Fall (1) ist an den steilen, vom Seegang senkrecht oder unter einem nicht $\mathrm{zu}$ flachen Winkel angelaufenen Felswänden gegeben. An diesen stehen die Flächen der Tierstöcke horizontal, das Medium pendelt senkrecht. Der nächsthäufigste Fall (2) ist realisiert, wenn solche Wände zu einem horizontalen Sockel einschwenken. Die Stöcke stehen dann senkrecht, ihre Flächen parallel zur Küste, das Medium pendelt horizontal und normal zur Küste. Seltener beobachtet man (Fall 3), und zwar in jenen Schluchten, deren Mauern der Seegang fast nur parallel entlangzustreichen vermag, daß die Flächen der horizontal wachsenden Stöcke verwirbelt stehen. Die kreisende Teilchenbewegung bleibt an diesen Stellen weitgehend erhalten. Schwenken nun solche Wände (4) in zureichend breite, ebene Felsböden um, dann stehen die Fiedern wohl wieder senkrecht, aber mit den Flächen normal zur Küste, der Wasserkörper pendelt in diesem besonderen Fall parallel zur Küste.

Mit diesen Merkmalen ist eine, durch harmonisch pendelndes Waser gekennzeichnete $\mathrm{Schwingungszone} \mathrm{abgrenzbar,} \mathrm{die} \mathrm{in} \mathrm{einer} \mathrm{Tiefe,} \mathrm{welche} \mathrm{der} 2^{1 / 2}$ fachen Wellenhöhe entspricht, beginnt (nach Ozeanographie und Wasserbau; Ort der zweiten Grundberührung der Wellen). Sie reicht bis in eine Tiefe, die, gewöhnlich der halben (Ort der 1. Grundberührung) bis knapp der ganzen Wellenlänge entsprechend, die Teilchengeschwindigkeit der Pendelbewegung unter jene der Streichbewegung sinken läßt. Die biologische Schwingungszone umfaßt somit etwas mehr als den verengten Schwingungsraum der Hydrodynamik (der mit der ersten Grundberührung beginnt), schließt aber den Raum der eigentlichen Brandung (im Felslitoral meist die Klippen- 
brandung) aus. Die Obergrenze verschiebt sich mit der Wellenhöhe, die Untergrenze jedoch nach dem Verhältnis von Wellenlänge und der Geschwindigkeit des streichenden Wasserkörpers (s. Seite 167). Staudruck, Durchflußmenge und Teilchengeschwindigkeit erreichen hohe Werte, der Rhythmus ist harmonisch und bleibt in der SekundenGrößenordnung, die Wasserversetzung ist vergleichsweise gering und erfolgt zudem vertikal sowie senkrecht auf die Küste.

\section{Das Zerreißen schwingender Wasserkörper}

Die biologische Brandungszone umfaßt jenen Abschnitt, in welchem die anlaufenden Wogen über zu flach werdendem Grunde überkippen und zusammenstürzen, oder an steilen Wänden direkt zerschellen (Strand- und Klippenbrandung). Kennzeichnend für dieses Gebiet ist das Aufplatzen der harmonischen Orbitalschwingungen, das Zerreißen der Wellenbäuche, die Durchmischung von Luft und Wasser. Die Bewegungsrichtung ändert sich zudem dauernd mit Wasserstand und Wellenhöhe. Die innere Brandungszone unterhalb des Ruhewasserspiegels besitzt dabei eine Tiefencrstredkung von rund der $2 \frac{1}{2}$ fachen Wellenhöhe, die äußere Brandungszone kann sich noch beträchtlich höher über den Ruhewasserspiegel erheben.

Im Vergleich zur Begriffsabgrenzung der Ozeanographie und des maritimen Wasserbaues handelt es sich hier um den Brandungsbereich im engsten Sinne. Auch das Gebiet der sog. Restwellen, das hinter dem engeren Brandungsbereich und überall dort auftritt, wo die Brandungsterrasse beträchtlich vor der eigentlichen Küste liegt (bei ausgedehnten, vorgelagerten Untiefen und Riffkanten) ist dem Brandungsbereich im biologischen Sinn nicht einzurechnen. Staudruck, Durchflußmenge und Teilchengeschwindigkeit erreichen hier maximale Werte. Ausgehend von der Geschwindigkeit der ungestörten Orbitalbewegung zwischen 40 und $200 \mathrm{~cm} / \mathrm{sek}$ können losgerissene Wasserteilchen bis zu 1000 und $7000 \mathrm{~cm} / \mathrm{sek}$ erreichen. Die sehr häufige Durchmischung von Wasser und Luft stellt einen weiteren Extremfaktor dar. Die Wasserversetzung dagegen ist wiederum kaum erhöht und damit gegenüber den übrigen Kräften ganz beträchtlich zurückgefallen.

Biologisch grenzt sich der Brandungsbereich durch den Wegfall einer großen Zahl von Arten ab, die entweder die hohen Staudrucke und die häufige Luftberührung nicht vertragen, die sich auf Grund der Deckungslosigkeit des Gebietes nicht behaupten oder im schießenden Wasser nicht ernähren können. Auffallend ist die Selektion schildförmiger kalkgepanzerter Formen, Chtbamalus stellatus und depressus, Patella coerulea und lusitanica, Spirorbis pagenstecheri, Chiton olivaceus, Middendorfá caprearum, Verruca stroemia und Haliotis lamellosa (in der Reihenfolge abnehmenden Trockenfallens aufgezählt), die, offenbar auf Grund in der Folge aufgetretener Anpassungen, das Brandungsgebiet auch kaum mehr verlassen.

Die im Brandungsbereiche auftretenden Kräfte sind im allgemeinen noch schwerer vorauszusehen als jene der beiden anderen Gebiete. Das kommt, wie die Molenberechnungen zeigen, daher, daß eine ganze Reihe komplexer Faktoren jeweils starken Einfluß nimmt. Neben der Höhe und Steilheit der anlaufenden Wellen und deren Periode kommt es auf den Aufriß des Küstenprofiles, die Art der Verengung des Schwin- 
gungsraumes, an (die Molenberechnung betrachtet die Teile des Profiles, Wassertiefe in Strandnähe, Molen-Fußvorlage und Molenform getrennt). Die Art der Energiekonzentration und die des Auftreffens der dynamischen Kräfte auf den Widerstand sind dabei von entscheidender Bedeutung. Selbst Einzelheiten der Form sind von. Wichtigkeit. Hohlräume und Uberhänge im Brandungsbereiche lassen, wenn auch nur Bruchteile von Sekunden lang, durch das Einpressen und explosionsartige Entweichen von Luftaschen durch die anstürmende, überbrechende Woge besonders hohe Belastungen auftreten (Irbarren 1953, Domzig 1955 Abb. 5 u. 6). Die vielfach ausgebildete Brandungshohlkehle (Cvijić 1924, GuILCHer 1945) gibt dazu ständigen Anlaß und ist in ihrer Entstehung selbst wieder zum Teil daraus zu verstehen. Nicht minder spielt im natürlichen Felsgebiet der Grundriß der Küste eine Rolle. Im Molenbau vermeidet man alle größeren Einziehungen, weil sie ein Bildungsherd neuer Energiekonzentration wären, und studiert ihren Effekt weniger eingehend. An der natürlichen Felsküste treten dieselben aber immer wieder und sehr wirkungsvoll in Erscheinung. Schließlich ist noch die Anlaufrichtung der Wogen einzurechnen. An senkrecht-glatten, tiefreichenden Felsmauern von. Buchtflanken können die schwersten Seen, so sie parallel einlaufen, nahezu reibungslos vorbeiziehen, um erst im Hintergrund der Bucht ihre noch volle Energie zu zerstören. Und endlich ist noch zu bedenken, daß die jeweils speziellen Verhältnisse von Energiekonzentration und Lage des Widerstandes mit den Wasserständen laufend wechseln.

\section{DIE EXPOSITIONSGEFALLE}

Wie nun bereits zu erwarten, sind auch die biologischen Bedingungen der Exposition nicht nach dem Schema der Ozeanographie, der maritimen Geologie oder Bautechnik zu gliedern, obwohl in eben diesen Disziplinen wiederum alle jene hydrodynamischen Fakten enthalten sind, auf welche wir uns derzeit stützen können. Hier sind jene Bewegungsgefälle als Grundeinteilung verwendet, welche durch die Küstenlage, die Wassertiefe, die Substratgestalt und die Substratgliederung bestimmt werden.

\section{Bedingungen der Küstenexposition}

Der Expositionsgrad einer Küste wird fast ausschließlich von jenen Kräften bestimmt, die mit der Bewegung des schwingend-zerreißenden Wasserkörpers auftreten. Die Lage der Küste gegenüber den örtlichen Seegangsverhältnissen ist darum von ausschlaggebender Bedeutung. Die Stärke des Seeganges wiederum ist, nach ozeanographischer Erfahrung, von der Häufigkeit, der Dauer und Stärke der örtlichen Winde und deren Streichlänge über das vor der Küste liegende Meeresgebiet abhängig. Wären diese Größen im jeweiligen Litoralgebiet zureichend bekannt, so könnte man die Formen des Seeganges, der im Laufe eines Jahres auf eine Küste zukommt, voraussagen. Direkter noch ließen sich die ankommenden Kräfte abschätzen, wäre Häufigkeit, Dauer und Richtung des jährlichen Seeganges mit seinen Wellenhöhen, -längen und -perioden kontinuierlich aufgezeichnet worden. Aber - und darunter leiden meist 
schon die Berechnungsansätze der Wasserbauer - auch über solche Unterlagen pflegt man nur ausnahmsweise zu verfügen. Dazu kommt, daß selbst diese Kenntnisse für den Biologen noch nicht reichten, denn was von den entfalteten dynamischen Kräften tatsächlich bis zu den Sedentariern der Küste kommt, hängt - nach der Erfahrung der Molen- und Dammberechnungen - wiederum von Auf- und Grundrißprofil der jeweiligen Küstenstelle $a b$.

Der Biologe vermag eine Vorstellung von der Verteilung der Kräfte gewinnen, wenn er entlang des Küstenverlaufes das Auf- und Absteigen der Obergrenze der sog. schwarzen und weißen Zone des Supralitorales verfolgt (siehe auch Abb. 9). Die schwarze Zone ist bekanntlich durch den Besatz der Felsen mit endolithischen Cyanophyceen (meist Hyella-, Mastigocoelus- und Calotbrix-Arten) und die Strandschnecke Littorina neritoides, die weiße Zone durch Flechten (Caloplaca, Lichina, Verrucaria) und Halophyten (Statice cancellata, Artbrocnemum glaucum, Critbmum maritimum und andere) zwischen Flächen blanken Felsens, gekennzeichnet. Die Anordnung der Sedentarier im obersten Teile des Sublitorales läßt die Expositionsverhältnisse noch genauer erkennen, nämlich sowohl deren Abstufung als auch die Größenordnung des Gefälles ablesen.

Die Abstufung: Es hat sich nämlich herausgestellt, daß die am Aufbau der Hydroidenbestände der Höhlen beteiligten Arten ihre Verbreitungsbänder nach dem Expositionsgrad und in gleichbleibender Reihe abfolgen lassen (RIEDL 1959). Mit abnehmendem Expositionsgrad rücken die 7 Hydroiden-Zonen immer meht aus den Höhlen heraus, so daß sich an den Eingangsrändern jeweils Zonen mit geringerem Expositionstypus (also mit höherer Ziffer) befinden. Entsprechend kann man den Expositionsgrad der einzelnen Küstenabschnitte nach dem dieser 7 stufigen Abfolge bezeichnen. Der größte Teil der geraden Küstenabschnitte um das "Capo di Sorrento" (Abb. 4) ist durch die gut exponiert stehenden Arten der Zone 2-3 und 3, durch Dynamena cavolinii und Aglaophenia pluma f. typica und f. octodonta gekennzeichnet, die Mehrzahl der Kaps durch die stärker exponiert stehende Zone 2 mit Aglaophenia pluma f. octodonta allein, nur an der sehr exponierten Punta-Spinosella etwa finden sich größere Bestände von Aglaophenia septifera der Zone 1. An den Buchtflanken stehen die für die Zonen 4 und 5 charakteristischen Arten Thecocaulus diaphanus, Plumularia setacea, Laomedea flexuosa und Halecium mediterraneum. Im Buchtinneren folgen die Zonen 6 mit Gonotbyraea gracilis und Tubiclava fruticosa, schließlich nahe den Felslitoral-Enden Nr. 7 mit Campanularia alta. Die Übereinstimmung dieser Vertelung mit dem Offnungswinkel der Küstenabschnitte, der Steilheit des Profiles und einem wahrscheinlich vorherrschenden Wellenanlauf aus der Generalrichtung Nordwest ist nicht zu verkennen.

Die Gefällegröße: Besitzen wir auch noch keine sichere Handhabe, um das Expositionsgefälle entlang der Küstenlinie quantitativ abschätzen zu können, so kann man sich doch jener fallenden Werte bedienen, welche mit zunehmender Wassertiefe, also bei einfacherer Faktorenabhängigkeit, viel greifbarer sind (RIEDL 1964b). Die Geschwindigkeit der kreisenden, in Bodennähe pendelnden Wasserteilchen reduziert sich bei einem Seegang von $0,25,1$ und $2 \mathrm{~m}$ Wellenhöhe in jeweils 8,12 und $20 \mathrm{~m}$ 'Tiefe um jeweils gerade eine Dezimale (vgl. den folgenden Absatz). Überprüft man nun die Veränderung der Hydrozoenzone, etwa unterhalb einer Küstenstelle vom 
Typus 1, an den Höhleneingängen einer Vertikalreihe, so stellt man fest, daß nach einem Bewegungsgefälle von einer Dezimale 4 Hydroidenzonen durchlaufen worden sind (Zonen 2 bis 4 in 2 bis $7 \mathrm{~m}$, Zonen 6 und 7 ab 20 und 30 m Tiefe; siehe auch Abb. 10). Legt man den so gewonnenen Maßstab an die Küstenreihe, so ergibt sich ein Gesamtgefälle von rund 11/2 Dezimalen, beziehungsweise ein mittleres Gefälle von Zone zu Zone in der Größe ron $2 / 10$ einer Dezimale.

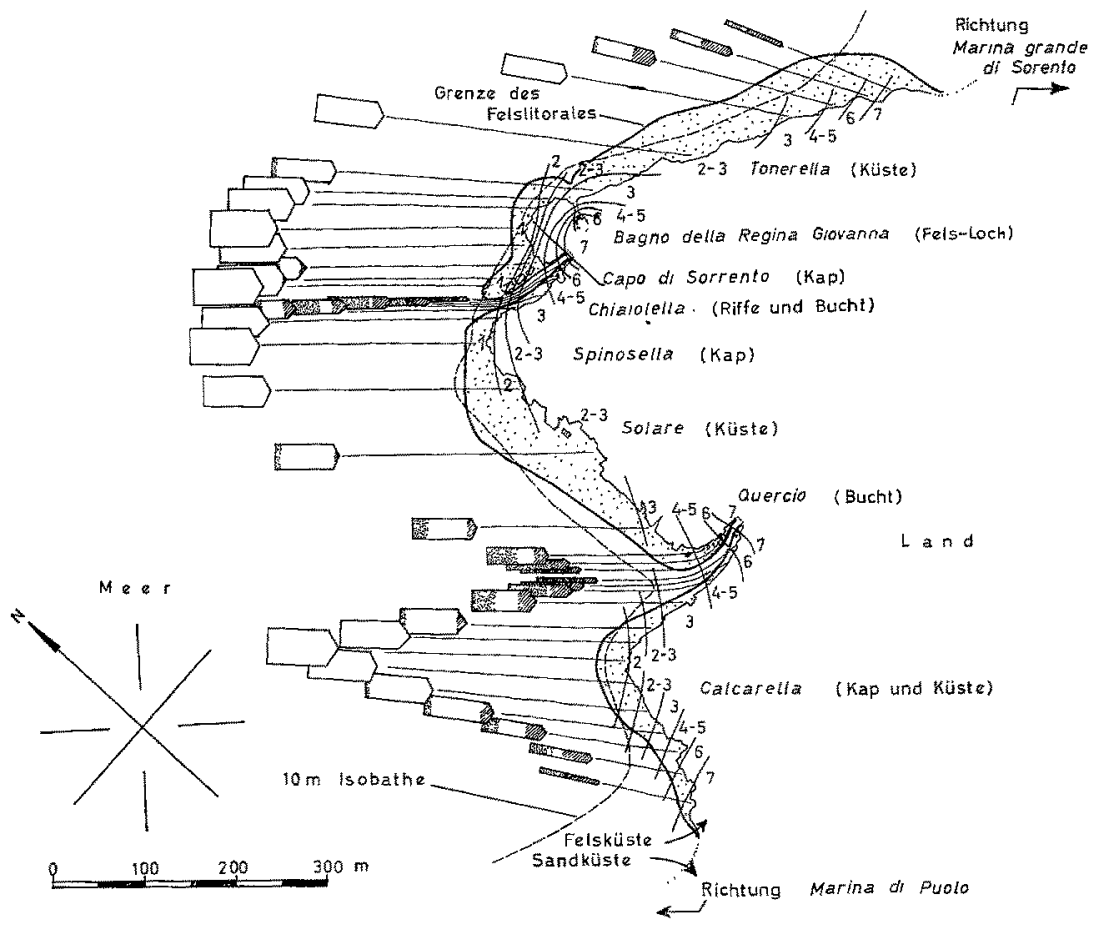

Abb. 4: Die Wirkung der Küstenexposition auf die Anordnung der Hydroiden-Zonen (der Felslitoral-Schattengebiete); am Beispiel der Küste zwischen der Marina grande di Sorrento und Puolo, im Golf von Neapel. Sublitorale Felsböden punktiert. Entlang der Küste sind die Hydroidenzonen 1 bis 7 und die Lage ihrer Obergrenzen eingetragen. Aus diesen abgeleitet geben die Pfeile Richtung und Stärke der Expositionen an. Die punktierten Teile der Pfeile geben die Expositionsschwächung durch flaches Küstenprofil, die gestrichelten Teile jene durch den Abschirmwinkel an

\section{Exposition und Wassertiefe}

Schon bei der Erörterung der Bewegungsweisen waren drei, sich am Seegrund unterschiedlich verhaltende Wasserkörper zu unterscheiden gewesen, welche mit zunehmender Tiefe einander ablösen. Man kann darum von drei Zonen der Wasserbewegung sprechen, was den Vorteil hat, die Veränderung der Exposicionsverhältnisse des ganzen Profiles nach den Vorgängen in jeder der einzelnen Zonen und jenen an den Zonengrenzen, den "kritischen Tiefen“ beurteilen zu können. Dabei ist gleich festzustellen, daß nur in der mittleren, in der Schwingungszone, Bedingungen herrschen, die ein 
beträchtliches Bewegungsgefälle verursachen. In der Brandungszone muß ebensowenig ein Gefälle ausgebildet sein, wie in der Strömungszone. In der Schwingungszone hingegen sinkt die Teilchengeschwindigkeit gesetzmäßig und verliert sich gegen die Tiefe völlig. Durch das Kleinerwerden der Radien der Orbitalbewegung bei zunehmender Tiefe verringert sich $\mathrm{z}$. B. bei mäßig langen, $1 \mathrm{~m}$ hohen Wellen die Geschwindigkeit der Wasserteilchen von einem Ausgangswert von rund $80 \mathrm{~cm} /$ sek gegen 2 und $20 \mathrm{~m}$ Tiefe auf 50 und $0,5 \mathrm{~cm} / \mathrm{sek}$.

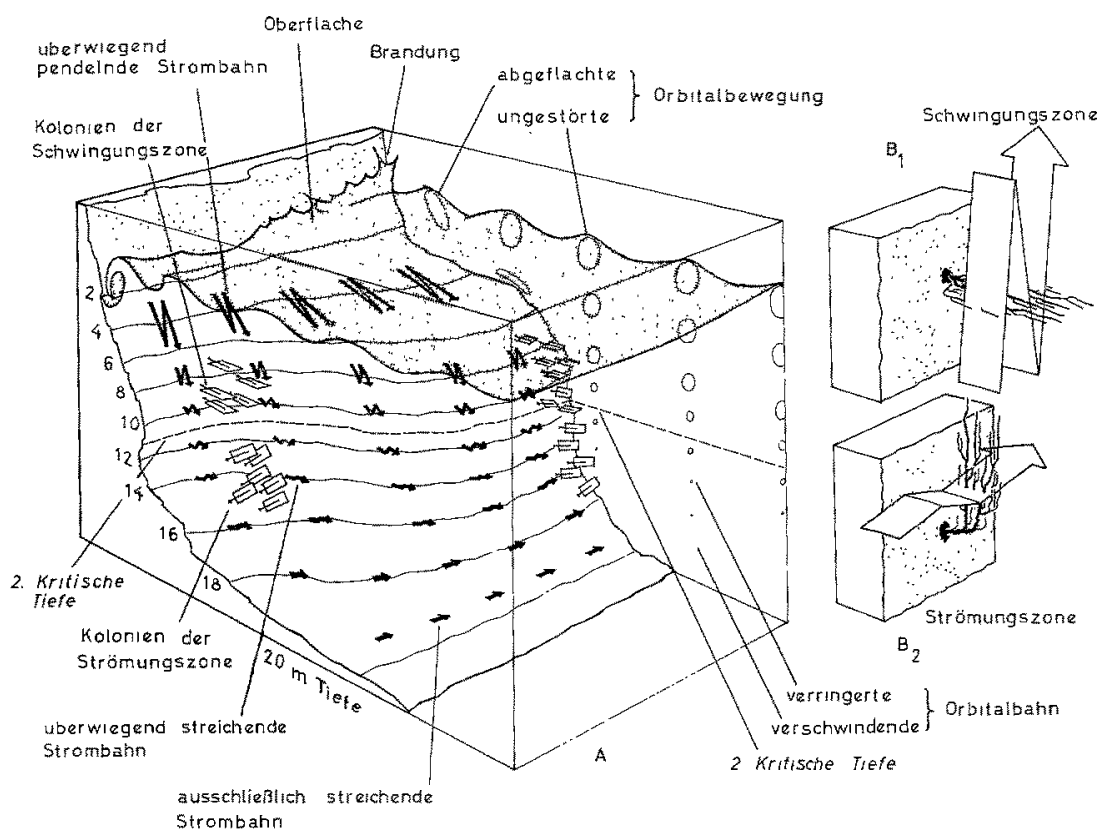

Abb. 5: Die Wirkung der 2. kritischen Tiefe, zwischen pendelndem und streichendem Wasserkörper, auf die Orientierung der großen und flächigen, passiven Filtrierer. $A$ Ubberhöhtes Blockdiagramm eines Küstenausschnittes von etwa $50 \mathrm{mal} 70 \mathrm{~m}$ und $20 \mathrm{~m}$ Tiefe. B1 Ausschnitt oberhalb der 2. kritischen Tiefe; $B 2$ unterhalb derselben. Die gestielten Rechtecke in $A$ und die Figuren in $B$ stehen für Anthozoen von flähigem Wuchs (Ennicella cavolinii und z. T. Eunicella stricta). Die Pfeile geben die aus Pendeln und Streichen resultierenden Bahnen der Wasserteilchen im Laufe von $1 \frac{1}{2}$ Orbitalperioden an. Beachte die verwirbelte Stellung der Kolonien und die Teilchenbewegung in der 2. kritischen Tiefe (gestrichelt). (Zum Teil nach RIEDL 1964a)

Die beiden kritischen Tiefen sind in dem fast gezeitenlosen Mittelmeer in ihrer Tiefenlage von der Wellenhöhe, der Wellenlänge und der Stärke der Küstenströmung abhängig.

Die erste kritische Tiefe, der Übergang vom zerreißenden zum harmonisch schwingenden Wasser hängt von der Wellenhöhe $\mathrm{ab}$ und befindet sich bei $1 \mathrm{~m}$ hohen Wogen in rund $2,5 \mathrm{~m}$ Tiefe. In sehr geschützten Gebieten steigt sie fast ganz an die Oberfläche und bringt damit die Brandungszone zum Verschwinden.

Die $z$ weite $k$ ritische Tiefe, der Ubergang von der überwiegend schwingenden zur vorwiegend strömenden Bewegungsform befindet sich bei einer Wellenlänge 
von 4 sek (Durchstreichzeit der Woge von Wellenberg zu Wellenberg) und einer örtlichen Strömung von $10 \mathrm{~cm} / \mathrm{sek}$ in einer Tiefe von rund $11 \mathrm{~m}$. Die bathymetrische Lage hängt dabei von der Wellenlänge und der Strömung in dem Maße ab, als die kritische Tiefe bei zunehmender Strömung weiter gegen die Oberfläche, bei zunchmender Wellenlänge weiter gegen die Tiefe verschoben wird. Der Ubergang befinder sich dort, wo die Geschwindigkeit der Pendelbewegung, welche mit der Tiefe abnimmt, auf den Wert der örtlichen Strömungsgeschwindigkeit gesunken, die Teilchenbewegung in beiden Hauptrichtungen gleich groß geworden ist (Abb. 5).

Man erkennt aus diesen Zusammenhängen, daß die Tiefenlage der ersten kritischen Zone mit dem Wechsel der Seegangsverhältnisse, die der zweiten zudem durch den Wechsel der Strömungsgeschwindigkeit an der Küste, laufenden Veränderungen unterworfen ist. Ihre mittlere Lage nach den örtlichen Bewegungsverhältnissen zu errechnen, ist schwierig. Um so wertvoller ist es darum, daß gerade die schwerer einsehbare 2. kritische Tiefe durch das Verhalten der Sedentarier sehr deutlich markiert wird. Die Hornkorallen Eunicella cavolinii und Eunicella stricta stehen nämlich in der Schwingungszone mit ihren Flächen horizontal (an Steilwänden) oder parallel zur Küste (an wenig geneigten Felsböden), in der Strömungszone hingegen stets senkrecht zum Küstenverlauf. Sie bieten damit der Haupt-Strömungsrichtung stets die Breitseite ihrer Flächen (ABEL 1959, RIEDL 1964a, 1964b). Nur auf der kurzen Stredke der 2. kritischen Tiefe vermögen sie sich nicht zu entscheiden, hier stehen ihre Flächen zueinander verwirbelt, vielfach $45^{\circ} \mathrm{zu}$ den vorwiegenden Strömungsrichtungen geneigt.

\section{Exposition und Substratgestalt}

Die Konfiguration der Substratoberfläche, des großen Reliefs, nimmt auf die Expositionsbedingungen insofern gleichmäßigen Einfluß, als an der Mehrzahl der Verengungen, welche sich der Wasserversetzung entgegenstellen, die Teilchengeschwindigkeiten zunehmen, innerhalb der meisten blindgeschlossenen Felsformationen und der Erweiterungen aber die Geschwindigkeiten sinken. Rechnet man die vorwiegenden Bewegungsrichtungen, z. B. in den beiden hier am meisten interessierenden Zonen, in der Schwingungszone und in der Strömungszone des Decliviums ein, so stellt man fest, $\mathrm{da} ß$ in der Schwingungszone hauptsächlich die Gesimse, in der Strömungszone die vorspringenden Mauerkanten zu Beschleunigungen führen. In beiden Zonen dagegen führen sackförmige Vertiefungen und Höhlen zum Sinken der Bewegung.

Das Studium der Hydroiden-Anordnung und der Stellung ihrer flächigen Repräsentanten haben (RredL 1959) Aufschluß über diese Anderung der Bewegungserscheinungen gebracht. Besonders die komplizierten Konfigurationen der unterseeischen Höhlen haben Anlaß gegeben, diese Verhältnisse kennenzulernen (ausführlich in RiEDL 1964b). Je nachdem die Höhlen einen oder mehrere Eingänge besitzen, ob sie die Wasserlinie schneiden oder nicht, entstehen hydrodynamisch ganz verschiedene Bedingungen. Zudem hat es sich gezeigt, daß die einzelnen Teile eines Höhlensystems, nämlich die Hallen, Stollen, Schächte, die Korridore, Nischen und blinden Gänge, wiederum nach dem Schema „Eingangszahl und Lage zur Wasserlinie“ jeweils unterschiedliche Konditionen schaffen. Sie verhalten sich wie die verschiedenen Höh- 
lentypen selbst. In zusammengesetzten Höhlensystemen entstehen dadurch besonders komplizierte Verhältnisse. Die Anordnung der Hydroiden ist jedoch so konsequent, daß man aus ihr die Strömungskarten der einzelnen Höhlen abzulesen vermag.

Die Gegenüberstellung einer Sadkhöhle (Höhle Ost/21) und eines mäßig großen Höhlensystems (Höhle Ost/39 der Tyrrhenia-Expedition/RIEDL 1959) möge zur Erläuterung der Verhältnisse dienen (Abb. 6). Die Hydroidenzonen 2 (bzw. 3) bis 7 geben dabei über die mittlere Stärke der Bewegung Aufschluß, die Orientierung der flächigen Stöckchen dagegen über die Richtung.

Höhle Ost/21 ist sadkförmig, der Eingang schneidet die Wasserlinie nicht, der Expositionsgrad des Küstenabschnittes entspricht der Hydroidenzone 3. Die Aglaophenia-Stöcke finden sich im Eingangsgebiet, ihre Flächen stehen parallel zur Ein-

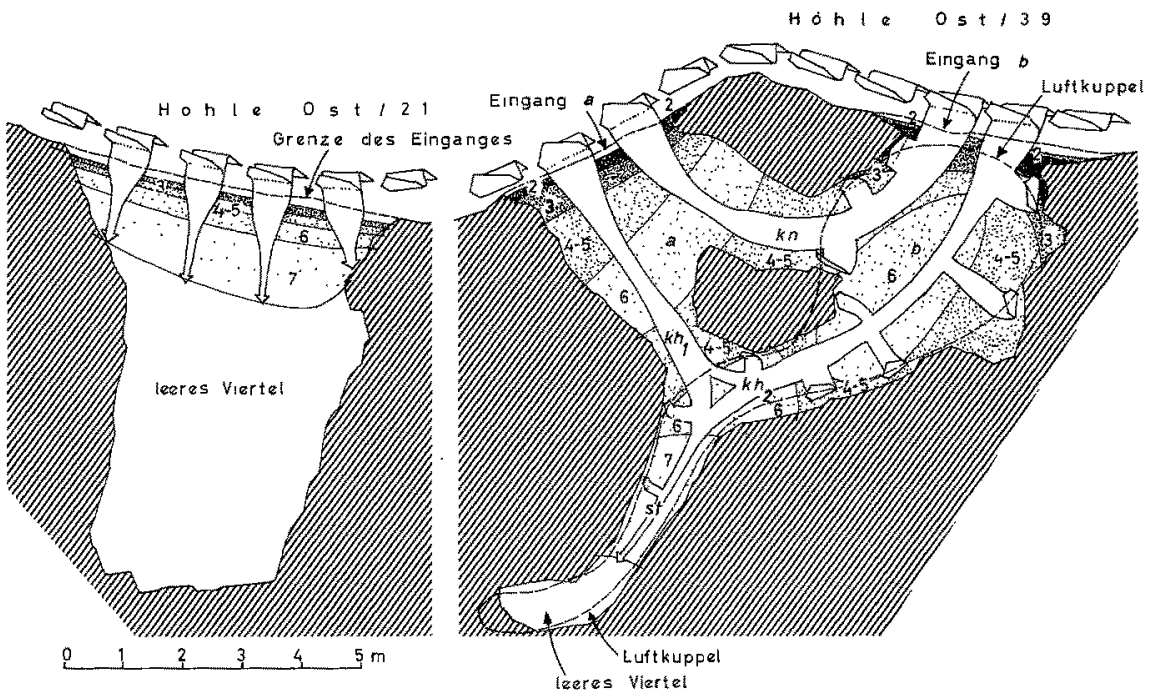

Abb. 6: Die Wirkung der Substratgestalt auf das Expositionsgefälle, dargestellt am Beispiel der Fydroidenzonen zweier Höhlen. Beide Höhlen im Grundriß (gesdinitten gedachter Fels sdiraffiert, Hydroidenzonen (2-7) abgestuft punktiert, leeres Viertel weiß). Die Pfeile geben die Strömungsrichtung, ihre Breite deren Stärke an; aufgestellte Pfeilenden deuten auf überwiegend vertikales Pendeln hin (Stärke und Richtung sind der Anordnung und Orientierung der Hydroidenbestände entnommen)

gangsebene. Das Wasser pendelt an den Höhlenwänden parallel zur Unterlage und in der Richtung der Höhlen-Hauptachse (und zwar jeweils gegenläufig). Die Zone 3 ist nur wenige $\mathrm{dm}$ tief gestaffelt, Zone $5 \mathrm{kaum}$ ausgebildet, die Zonen 6 und 7 enden bereits nach 1 bis $11 / 2 \mathrm{~m}$, der restliche Höhlenhintergrund (obwohl noch $5 \mathrm{~m} \mathrm{lang}$ ) ist unbesiedelt, schlammbekleidet und umfaßt das „leere Viertel“. Die Bewegung wird gegen die Höhlentiefe gleichmäßig und rasch gebremst. Höhle Ost/39 ist ein System aus zwei Eingängen, den Hallen $a$ und $b$, einem niederen $(k n)$ und zwei hohen $(k b)$ Korridoren sowie einem blind endenden Stollen; zudem kompliziert dadurch, daß die Halle $a$, der Korridor $k h 2$ und der Stollen st die Wasserlinie schneiden. Der Expositionsgrad des Küstenabschnittes entspricht der Hydroidenzone 2. Die flächigen Hydroidenstöcke stehen in den ganz untergetauchten Teilen sämtlich normal zu den 
Hauptachsen der Höhlenteile, in den halb untergetauchten Teilen aber nahe der Oberfläche auch parallel zu dieser. Das kommt daher, daß in den Teilen mit Luftkuppeln auch Spiegelschwankungen im Wellenrhythmus, also vertikale Bewegungen in Oberflächennähe auftreten. Die Gesamtintensität der Bewegung fällt in den Hallen zurück, und zwar vom Typ der Zone 2 auf Nr. 6. In den beiden ganz untergetauchten Korridoren ( $k n$ und $k b 1$ ) steigt sie durch Düsenwirkung wieder deutlich um eine Stufe, nämlich auf Zone Nr. 4-5 an. Erst im blind endenden Stollen folgt Nr. 7 und anschließend das leere Viertel.

Daß es sich dabei um die Mittelwerte der Exposition handeln muß, zeigt die Beobachtung (ABEL 1959, Ruedl 1959), daß im Stollen der Höhle Ost/39 bei starkem Seegang besonders rasche Teilchengeschwindigkeiten auftreten können. Bei mäßig bewegter oder ruhiger See kommt dagegen der Wasserkörper im Stollen fast gänzlich zur Ruhe. Weiter unten (vgl. Seite 177) ist auf die sehr unterschiedlichen Bedingungen, welche Mittel- und Extremwerte setzen, noch zurückzukommen.

\section{Exposition und Substratgliederung}

War eben festzustellen, daß die Stärke der örtlichen Wasserbewegung auf Grund der Substratgestalt über Strecken von Metern bis Dezimetern um Dezimalen verändert zu werden vermag, so kann die Substratgliederung solche Gefälle schon auf Strecken von Zentimetern bis Millimetern zeigen.

Besonders auffallend werden diese Verhältnisse in enggegliederten, festen Substraten, etwa in den Kalkalgenbänken des "Trottoir", in jenen der Brandung ausgesetzten Bänken von Vermetus, von Serpuliden und von Balanus (sowie in den toten Teilen der Korallenbänke der Tropen). Festigkeit und enge Porengliederung bewirken gemeinsam eine äußerst rasche Reduktion der Teilchengeschwindigkeit. So kommt es, daß nur wenige Millimeter unter Substratflächen, über welche die Brandung mit Geschwindigkeiten von einigen Metern in der Sekunde hinwegfegt, Organismen anzutreffen sind (nicht haptische Turbellaria, Nemertini, Nematoda und viele andere), welche bereits von Bewegungen in der Zentimeter-Größenordnung weggespiilt werden würden. Auch dichte Algenbestände und filzige Epiphyten-Oberzüge scheinen ähnlich extrem expositionsabschirmende Standortbedingungen zu schaffen.

\section{DIE EINFACHEN WIRKUNGSWEISEN}

Die große Vielfalt der Wirkungsweisen der Wasserbewegung auf die Sedentarier läßt sich am anschaulichsten nach drei Einteilungsprinzipien erklären. Zweien sind wir bereits begegnet. Einmal haben sich die Erscheinungen desselben Bewegungsvorganges selbst als sehr unterschiedlich erwiesen. Vom Staudrudk bis zur Wasserversetzung ergibt sich eine ganze Reihe grundverschiedener Wirkungen. Ein andermal sind differente Bewegungsweisen zu unterscheiden. In einer Reihe, vom drehend-streichenden Wasserkörper unterhalb des Decliviums bis zum Zerreißen schwingender Wasserkörper in der inneren Brandungszone, liegen, sich stufenweise ändernd, verschiedene 
Ausgangskonstellationen vor. Zudem ist aber noch ins Auge zu fassen, daß die Wasserbewegung über verschiedenste Umwege auch indirekt auf die Organismen wirkt; sei es durch die mechanische Beanspruchung des Substrates, sei es durch den Transport von Sedimenten, Gasen, Verbreitungsstadien oder Nahrungsobjekten.

Folglich ist mit einer Anzahl qualitativ ganz verschiedener Wirkungsweisen zu rechnen, gewissermaßen einzelnen Aspekten, wie sie auch immer gruppenweise, sich gegenseitig bedingend oder ausschließend, auftreten mögen. Dabei ist noch festzustellen, daß der einzelne Aspekt den Schwerpunkt seiner Wirkung den maximalen, mittleren oder minimalen Kräften verdankt, daß also in quantitativer Hinsicht wiederum verschieden wirkende Ausschnitte in Erscheinung treten müssen.

\section{Die Aspekte der Wirkungsweisen}

Bei der folgenden Schilderung der etwa 9 bisher zureichend deutlich gewordenen Aspekte sei auf eine Rangordnung verzichtet. Noch ist es ganz ungewiß, ob die primären Wirkungen jene auf kurzen oder langen Umwegen einflußnehmenden, die sekundären und tertiären, an Bedeutung überträfen. Auch würde eine solche Sortierung - jedenfalls beim gegenwärtigen Stande der Kenntnisse - eine künstliche sein. Gewiß ist lediglich, daß jeder der zu beschreibenden Aspekte seinen eigenen, über Gedeih und Verderb der Formen entscheidenden Einfluß nimmt. Je ein Beispiel möge das wieder illustrieren.

Die Festigkeit des Substrates wird von den Maxima der örtlichen Bewegung, namentlich dem Staudruck, kontrolliert. Entsprechend den örtlichen Expositionsverhältnissen verschiebt sich die Grenze des eben noch zureichend festen Substrates in weiten Grenzen. In sehr stark exponierten Brandungsgebieten sind es die mehrkubikmeter-großen Blödke, auf Böden unter schwach streichenden Wasserkörpern Schellpartikel der Millimetergröße, die entweder schon liegenbleiben oder eben noch in zu kurzen Abständen bewegt, versetzt und umgewendet werden. Die Blockhalden des Brandungsgitrtels bieten das übersichtlichste Beispiel (Abb. 7). Unter gleichmäßig mittleren Expositionsbedingungen entsprechen die Bestände auf Blöcken über der $1 / 2-\mathrm{m}^{3}$-Dimension weitgehend jenen des gewachsenen Felsens. Die Oberseiten weisen die typischen Phytalbestände, die Flanken Phytal-Schattenbestände, die Unterflächen (soweit sie freiliegen) ausgereifte Höhlenbestände auf. Das Substrat liegt entsprechend fast ein Jahr oder noch länger unbewegt. Auf Blöcken über der 20-Liter-Größe lassen sich wohl noch dieselben drei Bestandszonen unterscheiden, aber sie sind lückenhaft ausgebildet, und die seitliche Schattenzone beginnt zu verschwinden. Dieses Substrat erreicht keine ganzjährigen Liegezeiten mehr. Zu oft werden die nach oben gerollten sedentarierbestandenen Unterseiten von Algen überwuchert und erstickt. Die Zeit für die Ausentwicklung der reinen Sedentarier-Bestände beginnt zu kurz zu werden (Rützler 1963, Riedi 1964b). Grobes Geröll über der 2-Liter-Größe läßt nur mehr die Oberseiten als einigermaßen differenziert besiedelt erkennen. Rasch aufwachsender Algen-Anflug macht sie kenntlich. Die wenigen jungen oder kleinen Sedentarier sind schütter über den ganzen Block verteilt. Die Liegezeit beträgt höchstens noch Monate. Geröll unter der Litergröße zeigt keine Differenzierung der einzelnen Seiten, und 


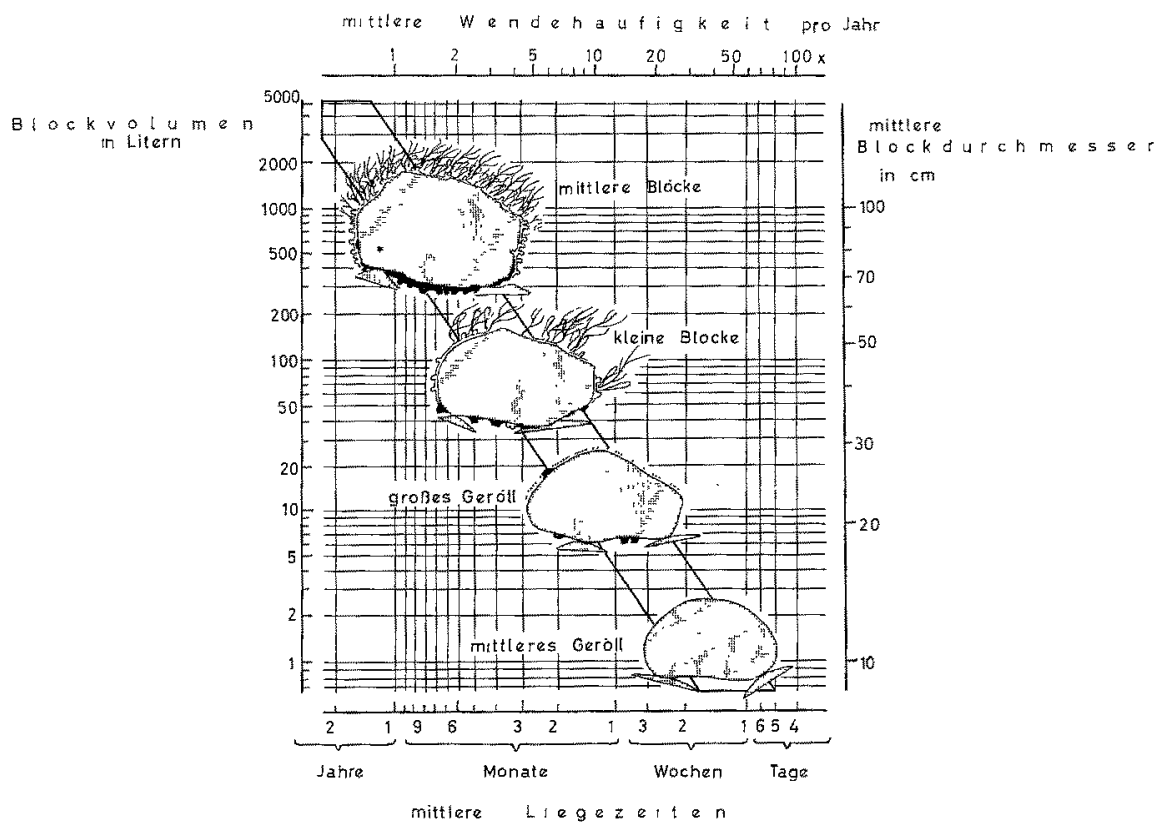

Abb. 7: Die Wirkung der Substratfestigkeit auf die Ausbildung von Sedentarier-Beständen. Mit abnehmender Blodkgröße steigt bei gleichbleibender Exposition im Mittel die Häuf́gkeit der Instabilität; die Liegezeiten werden zum Ausreifen zuerst der Bestände (Phytal ausgezeichnet, Schattenformation weiß̧, Höhlenbesiedler schwarz), danı ihrer Formen selbst zu kurz

außer endolithischen Formen finden sich keine Bestände mehr. Die Liegezeiten von nur mehr Wochen sind für fast alle Formen zu kurz geworden (Abb. 7).

Die Störung der Individuen durch Berührung, sei es durch Nachbarn oder flexible Substratteile, wird in erster Linie von der Teilchengeschwindigkeit, deren häufigen Mittelwerten, abhängen und vor allem in der Schwingungszone in Erscheinung treten. Besonders auf dichteren Algensubstraten kann man feststellen, daß die Hydroiden bei fortgesetzter Störung die Fangaktivität einstellen und vielfach die Hydranthen, sofern sie Theken besitzen, einziehen. In diesem Zusammenhang sei auf folgende auffallende Erscheinung verwiesen. Auf den flexiblen Substraten der Schwingungszone, so besonders auf Tangen, Seegras und Treibgut, siedeln mit einer Ausschließlichkeit, wie wir das von keinem anderen Lebensraume kennen, diejenigen Hydroiden, die tiefe Theken besitzen; athekate Formen und Haleciiden sind praktisch ausgeschlossen (RuEDL 1959 und 1964b). Ich möchte diese Erscheinung mit der Annahme erklären, daß die Theka einen Berührungsschutz bietet und verhindert, daß die Nesselbatterien bei den häufig anhaltenden Störungen fortgesetzt und nutzlos verschossen werden.

Die Resistenz der Individuen selbst wird von den Staudrucken kontrolliert, die bei hoher Teilchengeschwindigkeit, besonders in der oberen Schwingungszone und in der Brandungszone, auftreten. So fallen beispielsweise zarte, hochwüchsige Hydroiden schon in der Schwingungszone zurück; an stärker exponierten Stellen überwiegt 
der Niederwuchs oder aber die Formen ordnen sich zu Fittichen zusammen, die sich bei sehr schneller Wasserbewegung deckend zusammenlegen und dadurch ein strömungsableitendes System bilden. An schutzlos freiliegenden Substratflächen, die häufig unter schießendem Wasser liegen, verschwinden z. B. Hydroiden ganz. Die Sedentarier sind dann nur mehr durch Krustenformen vertreten. Unter sehr stark schießendem Wasser, etwa in extrem exponierten inneren „Brandungs-Düsen“ und BrandungsHohlkehlen, sind Sedentarier überhaupt nur mehr im Gestein selbst (durch Formen des Endolithion) repräsentiert.

Die Verschüttung durch Sediment-Zudrift ist an den Grenzen des Felslitorales, also in der Nähe mobiler Böden, besonders der leicht wandernden Sande, und bei anhaltender Wasserbewegung oft zu beobachten. Die Reaktion der Organismen ist gruppenweise sehr verschieden. Poriferen vertragen auch kurzzeitige Verschüttung schlecht, viele Formen vermögen aber auf sie fallendes Sediment, Sande, Schwammnadeln und sogar Steinchen in den Körper einzubauen. Verschüttete Hydroidkolonien versuchen zumeist durch Auswachsen der Stolonen abzuwandern. Aktinien gießen auch größere Mengen aufgefallenen Sedimentes durch Neigen und Wimpernschlag über einen Schleimstrom ab. Cirripedier und sedentäre Mollusken meiden solche Sedimentnähe. Manche Monascidien dagegen vertragen auffallend viel Sedimentaufschüttung.

Mangelnde Abdrift von Sediment, namentlich feinem Sediment, tritt bei anhaltend minimaler Wasserbewegung in Erscheinung. Nach längeren windlosen Perioden triff man Hydroiden, kleine stoloniale Anthozoen (Cornularia), Enthoprocta, Bryozoa und Synascidien of schlammüberzogen und in schlechtem Zustand an. Bei Anhalten des Zustandes werden $z$. B. die Hydranthen der Hydroiden ganz abgebaut. In unterseeischen Höhlen, in welchen die Besiedler der Böden viel häufiger von Verschlammung bedroht werden als jene der Decken, ist aufgefallen (RrEDL 1959), daß sich auf den Böden die Athecata scharen, an den Decken aber überwiegend Thecaphora siedeln, welche auf den Böden höchstens die vorspringenden Substrate besetzen oder auch diese meiden. Man gewinnt aus diesem Verteilungsverhalten den Eindruck, daß die Thecaphora, besonders Formen ohne Verschlußapparat, aber mit tiefen oder komplizierten Theken, eher von Verschmutzung bedroht werden als die Athekaten (und thekenlosen Thekaphoren).

Das Scheuern durch Bewegung von Grobsediment, namentlich von Schell und Geröll unter der Wirkung rasch pendelnder Wasserkörper, führt dort zur Bildung lebensfeindlicher Gebiete, wo Bodengestalt und Expositionslage der Erscheinung Dauer verleihen. Der Taucher erkennt sofort die bestandslosen, blankgeschliffenen Felsbahnen und -mühlen am Seegrund, auf deren Substrat sich nur einige wenige Formen des Endolithion zu halten vermögen. An reifen Arten des Epilithion finden sich nur winzige, raschlebige Arten, etwa Spirorbis pagenstecheri (Polychaeta) oder Lichenopera radiata (Bryozoa).

Die Ernährung durch Planktontransporthängt in erster Linie von der Größe der mittleren Durchflußmenge (gewissermaßen durch den Querschnitt des Sammelbereiches des jeweiligen Sedentariers) ab, in zweiter Linie auch von der Teilchengeschwindigkeit. Hohe Geschwindigkeit bestimmt z. B. bei Cirripediern die Filtermethode, die von der aktiven - bei schießendem Wasser - zur passiven Methode umschlägt (RIEDL 1964b). Sie führt bei manchen Hydroiden jedoch zum Einstellen der 
Aktivität, weil die vorbeischießenden Beuteobjekte offenbar nicht mehr gehalten werden können. Die Durchzugsmenge, in gewisser Hinsicht die Häufigkeit des Wasseraustausches, beeinflußt dagegen das Nahrungsangebot wesentlich. Die eminente Bedeutung des Wasseraustausches für die Ernährung der Sedentarier geht z. B. aus der aktiv gefilterten Wassermenge durch die Bestandsbildner der unterseeischen Höhlen hervor (RIEDL 1959, ausführlich 1964b). Es läßt sich errechnen (vgl. JøRGENSEN 1955), daß

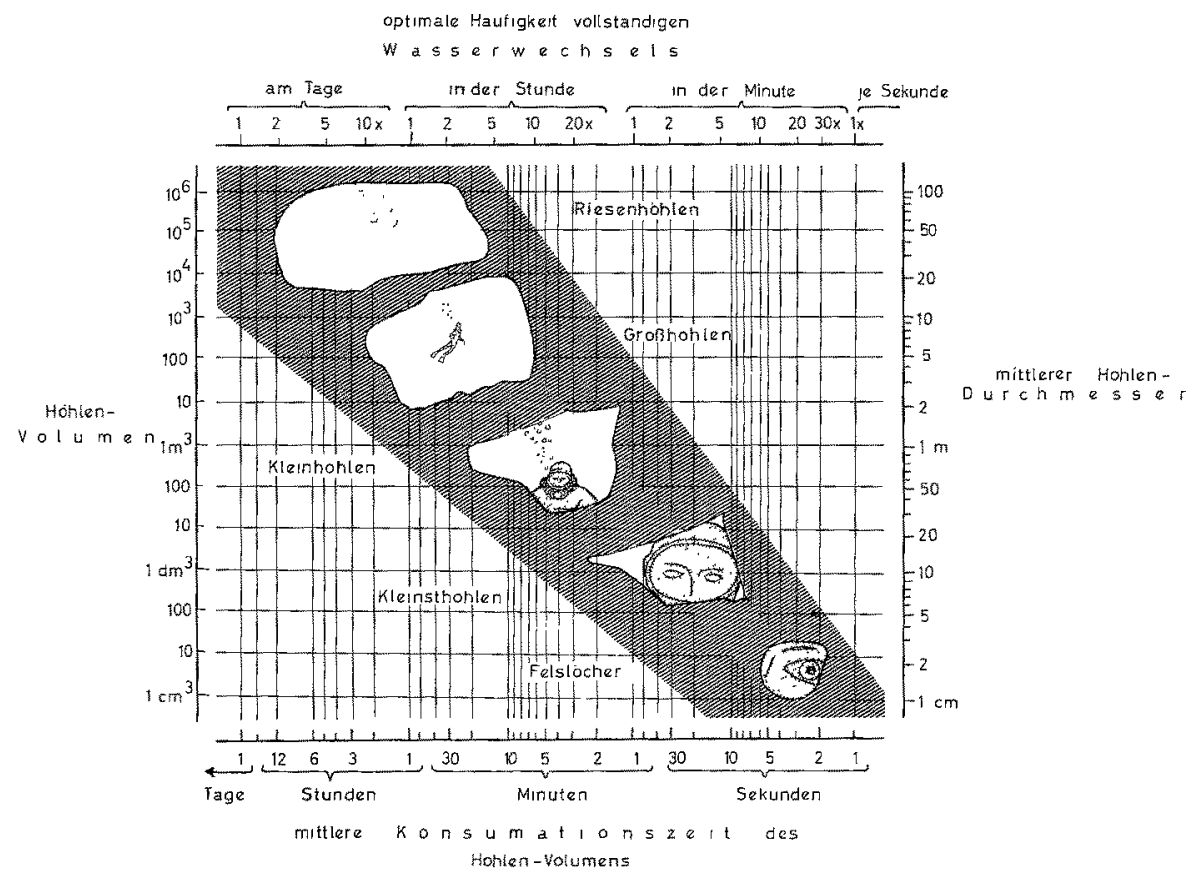

Abb. 8: Die Wirkung der Höhlengrößen auf die nötige Häufigkeit des Wasseraustausches zur optimalen Ernährung von Sedentarierbeständen mittlerer Dichte. Der Tabelle ist die errechnete Filtermenge von im Mittel 1,5 Tonnen Seewasser je Stunde und Quadratmeter Bestandsfläche zugrunde gelegt

Sedentarierbestände der üblichen Dichte das Wasser in Höhlen z. B. mit $1000 \mathrm{~m}^{3}, 1 \mathrm{~m}^{3}$ und 11 Volumen in etwa knapp einer Stunde, 10 Minuten bzw. einer Minute durchgefiltert haben. Um solchen Beständen optimale Ernährungsbedingungen zu sichern, muß das Höhlenwasser jeweils mindestens einmal, sechsmal bzw. sechzigmal in der Stunde ausgetauscht werden (Abb. 8).

Die Lüftung durch Transport von Gasen tritt in eine kritische Phase, sobald die Minima der Wasserversetzung zu lange anhalten. Bei begrenzten, stagnierenden Wasserkörpern tritt das besonders rasch in Erscheinung. Setzt man beispielsweise Poriferen, Hydroiden oder Bryozoen in eine Wanne mit stagnierendem Ortswasser, so werden - im Falle es sich um reife Individuen (bzw. Stöcke) handelt - auffallend rasch und in großen Mengen Larven und Medusen abgestoßen. Ich habe diese Vorgänge wiederholt bei Obelia- und Ircinia-Arten, bei Spongia officinalis und Scbizoporella san- 
guinea beobachtet. Zweifellos handelt es sich um eine Reaktion auf ungünstig werdende Klimabedingungen, welche den Sinn hat, im Fall klimatischer Bedrohung die Nachkommenschaft in Sicherheit zu bringen. Dabei läßt die Promptheit und Konsequenz des Vorganges vermuten, daß sich die Verschlechterung des Klimas noch kaum über die unmittelbare Wassersphäre um den Organismus hinaus ausgedehnt hat und im Beckenwasser selbst noch gar nicht zum Ausdruck kommt; daß also schon der Wegfall der Wasserbewegung den eigentlichen Auslöser darstellt.

Befruchtung und Verbreitung aller jener Formen, die Geschlechtszellen, Larven oder ganze Generationen ins freie Medium abgeben, und hierher zählen bekanntlich alle Sedentarier, werden durch das Ausmaß der Wasserversetzung maßgeblich beeinflußt. Dabei spielt sowohl die Teilchengeschwindigkeit, als auch die Durchzugsmenge eine ganz untergeordnete Rolle; wichtig ist die Strecke, die der Wasserkörper, welcher die freigewordenen Individuen aufgenommen hat, im Laufe ihrer freischwebenden Lebensperiode zurücklegt. Die Wichtigkeit dieses Aspektes der Wasserbewegung, für die Probleme der Artbildung und der Tiergeographie bedarf hier wohl keines weiteren Beleges. Ich darf mich hier mit einem Hinweis auf das schon gegebene Beispiel (Abb. 1) bescheiden.

\section{Die wirkenden Ausschnitte}

Im vorigen Abschnitt war festzustellen, daß die einzelnen Aspekte der Wasserbewegung vielfach mit einzelnen Ausschnitten einer Bewegungserscheinung entweder mit deren Maxima, Mittelwerten oder Minima zusammenhängen. So bilden beispielsweise bei den Aspekten Festigkeit, Resistenz, Verschüttung und Scheuern die Maxima, bei Säuberung, Lüftung und Verbreitung die Minima den gravierenden Ausschnitt. Daneben fanden sich aber auch schon Hinweise darauf, daß sich ein und derselbe Aspekt, beispielsweise der der Ernährung, bei mehr als einem wirkenden Ausschnitt entfalten kann, wobei wiederum verschiedene Einflüsse des Bewegungsklimas (gewissermaßen Unteraspekte) hervortreten. Die Schilderung solcher Unteraspekte sei mit den folgenden drei Beispielen versucht.

Die Maxima. Sobald man versucht, ein Bild von den an einem Standort auftretenden maximalen Bewegungserscheinungen zu gewinnen, stellt man fest, daß zuerst die Zeit festzulegen ist, innerhalb welcher das gesuchte Maximum verbindlich oder signifikant sein dürfte. Mit zunehmendem Zeitausschnitt werden die Extremwerte naturgemäß größer, keineswegs aber für den Einzelfall zutreffender. Die Signifi$\mathrm{kanz}$ einer Ausschnittslänge hängt viel mehr von der biologischen Erscheinung $\mathrm{ab}$, mit welcher sie in Beziehung gesetzt wird. Die Höhe des 24stündigen Maximums ist z. B. für die Bestimmung der Resistenz eines Cystoseira-Bestandes der Brandungszone ebenso unsignifikant wie die des 10jährigen. Im ersteren Fall muß man erwarten, daß die entscheidenden Maxima außerhalb des gerade beobachteten Tages liegen, im letzteren hingegen, daß die Zusammensetzung der Vegetation einer vielleicht vor Jahren durch eine Sturmflut zerschlagenen Kïste längst wieder ausgereifte Formen zeigt. Unsere Kenntnisse von langewährenden Erscheinungen im Sublitoral sind naturgemäß gering. Ich darf darum ein Beispiel aus dem freiliegenden Einflußgebiet des Meeres wählen, aus dem Supralitoral. 
Im felsigen Supralitoral des Mediterran pflegt man (wie schon erwähnt) eine untere schwarze und eine obere weiße Zone zu unterscheiden. Erstere ist durch eine Vegetation endolithischer Blau- und teils Grünalgen gekennzeichnet, letztere durch blanken, nur in Spalten humusführenden Fels, der nach oben von der Macchie (der

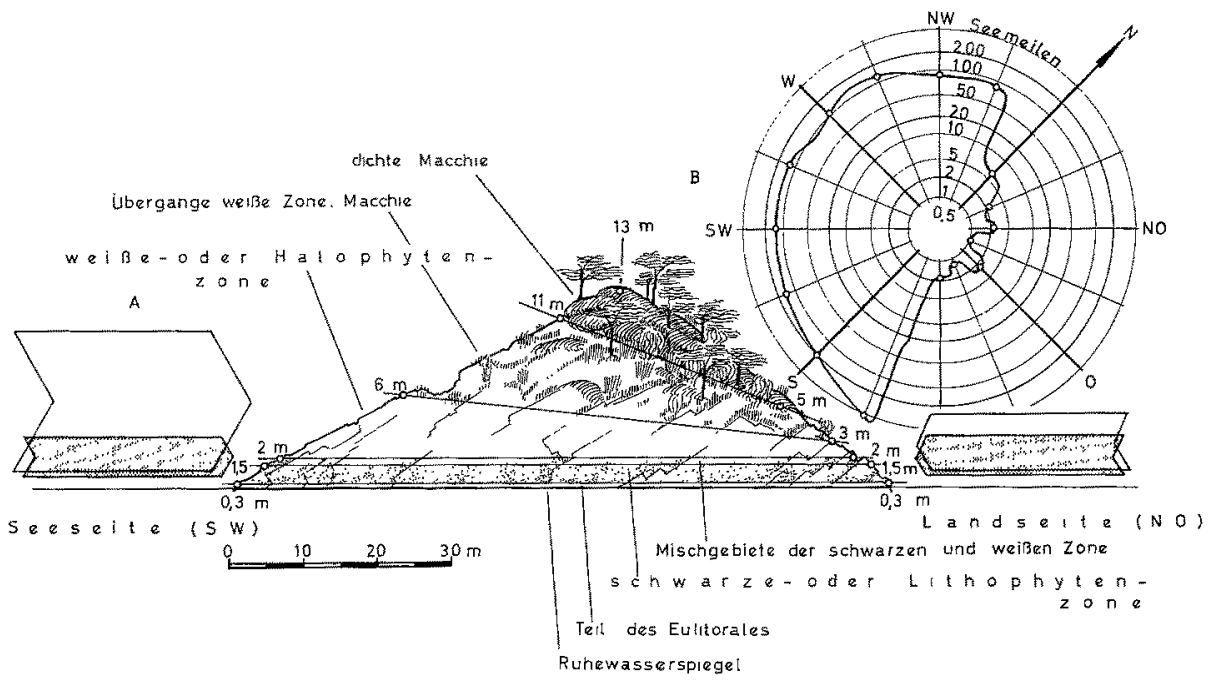

Abb. 9: Die Wirkung der Lang- und Kurzzeit-Maxima des Seeganges auf die Ausbildung der Zonen im Supralitoral. A Insel Banjole (Westküste Istrien) halbschematisch, zweifach überhöht, gesehen von Südost. Schwarze Zone punktiert, weiße Zone weiß. Die mittleren Seegangsstärken (Kurzzeit-Maxima, graue Pfeile) sind nach der Häufgkeit der Windrichtungen, die Maximalen (Langzeit-Maxima, weiße Pfeile) nach den Streichstrecken der Winde über See angegeben. $B$ Streichstrecken der auf Banjole zukommenden Winde über See

Decke des mediterranen Buschwaldes und seiner Ausläufer) begrenzt wird. Im Gefolge der Lithophyten erscheint die Strandschnecke Littorina neritoides, auf den salzkrustigen Felsen, Flechten und Halophyten, etwa Caloplaca aurantia und Statice cancellata. Man spricht darum auch von einer Litho- und Halophytenzone. Nun erheben sich die beiden Zonen ganz verschieden hoch über den Ruhewasserspiegel, entsprechend den allgemeinen örtlichen Expositionsverhältnissen. Aber ihr Auf- und Absteigen entlang der Küste erfolgt keineswegs im gleichen Rhythmus. Besonders eindeutig verhalten sich darin jene kleinen, der Küste nur wenig vorgelagerten Eilande, deren Flanken Seegang von ganz verschieden langer Anlaufstrecke empfangen. An der Insel Banjole beispielsweise (vor Rovinj an der Westküste Istriens) reicht die Lithophytenzone ringsum sehr gleichmäßig 1,5 bis $2 \mathrm{~m}$ über den mittleren Wasserspiegel. Die Halophytenzone hingegen (Abb. 9) reicht an der Landseite nur in 3 bis $5 \mathrm{~m}$ Höhe, an der Seeseite hingegen 6 bis 11 m hoch auf die Insel hinauf. Die Erstreckung der Lithophytenzone entspricht etwa den mittleren Seegangsverhältnissen, da es auf zureichend häufige Benetzung ankommt. Die Häufigkeiten der einzelnen Seegangsrichtungen rings um die Insel sind auch ziemlich ausgeglichen. Die Reichweite der Halophytenzone hingegen folgt den Maxima, da für deren Erhaltung ein weitgehendes Fortwaschen entstehenden Humus' auch in großen Abständen genügt. Die schweren, hohen Seen ent- 
falten sich aber nur über größere Anlaufstrecken (Streichlänge des Windes, vgl. Seite 164). Die kurze Entfernung von der Küste reicht für deren Bildung nicht aus. Anzufügen bleibt, daß im geschilderten Falle die sogenannten Mittelwerte, also die Werte jenes Seeganges, der zureicht, um die Lithophyten oft genug zu benetzen, exakter als Maxima kurzer Intervalle aufzufassen wären, denn es kommt nicht auf die

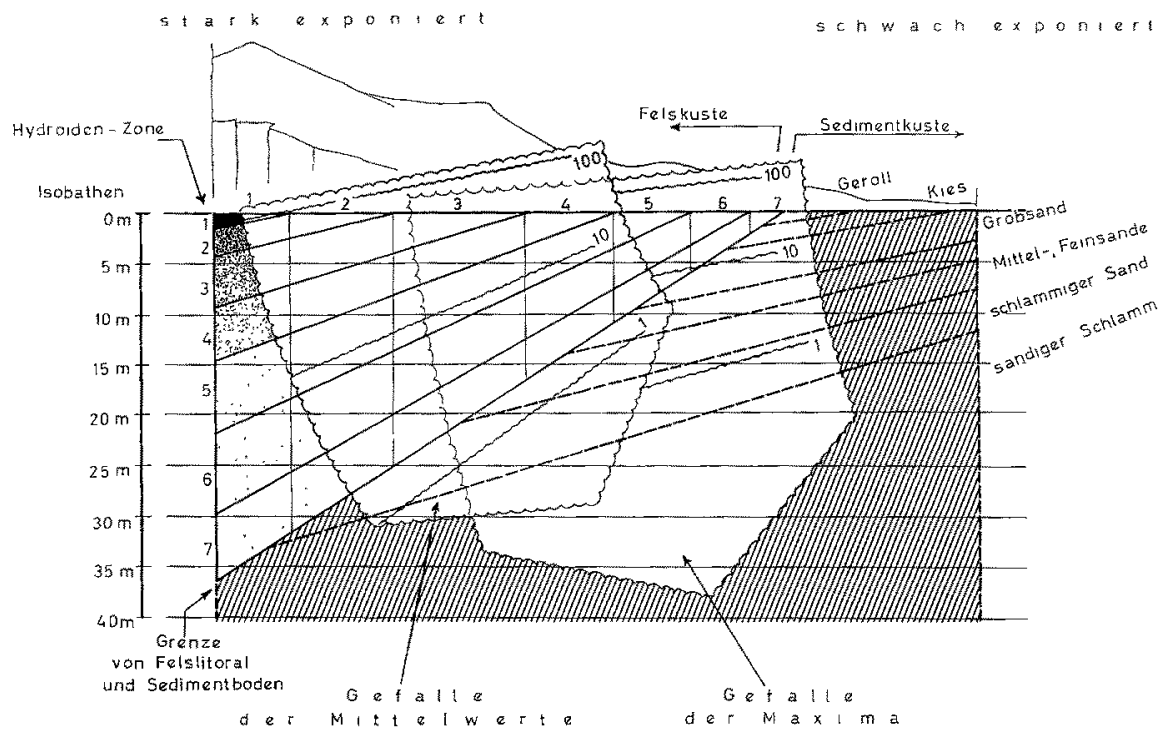

Abb. 10: Die Wirkung der mittleren und maximalen Expositionsgefälle auf die Verteilung der Hydroiden- und Sediment-Zonen; am Beispiel eines schematisierten, überhöhten Aufrisses des Sorrentiner Litoralgebietes (Golf von Neapel) von rund $1 \mathrm{~km}$ Länge, dargestellt bis $40 \mathrm{~m}$ Tiefe. Felslitoral gegliedert in die Hydroidenzonen 1-7 (abnehmend punktiert), Sedimentböden gegliedert nach Korngrößen (schraffiert). Die breiten Pfeile geben die Neigungen und die relativen Größen (Verlauf der Dezimalwerte gewellt) der Expositionsgefälle an. Das Gefälle der Mittelwerte ist aus der Anordnung der Hydroidenzonen (Grenzen ausgezogen), das der Maxima aus der Korngrößenverteilung (Grenzen gestrichelt) abgeleitet

Summe der Kräfte in einem Zeitabschnitt, sondern wiederum auf zureichend häufige Zwischen- oder Kurzzeit-Maxima an. Doch seien die Verhältnisse hier nicht weiter zerlegt, als sie bisher auch biologisch zu belegen sind.

Die Mittelwerte bilden in ihrer Abgrenzung ein doppeltes Problem. Einmal ist es wieder die Festlegung des signifikanten Zeitintervalles, ähnlich wie bei den Extremwerten. Zudem ist es aber - wie eben angedeutet - in der Regel schwierig, echte Mittelwerte von ganz ähnlich wirkenden Kurzzeit-Extremen (seien es nun Maxima oder Minima) zu trennen. Man sollte erwarten, daß echte Mittelwerte wahrscheinlich nur dort eine biologische Beziehung einrichten, wo in erster Linie die $\mathrm{Summe}$ von Kräften (schwankender Größen) eine biologische Rolle spielen. Derlei darf man etwa bei der Abhängigkeit der passiven Filtrierer (vgl. S. 157) von den Summen der Durchflußmenge erwarten.

Greift man beispielsweise auf die Kennzeichnung der Expositionsverhältnisse durch die sieben Hydroiden-Zonen der Felslitoral-Schattengebiete zurück, so war 
festzustellen, daß dieselben gegen die Ränder des Felslitorals, sowohl horizontal (mit abnehmender Küstenexposition) als auch vertikal (mit der Tiefenreduktion der Wasserbewegung) einandergereiht ablösen. Daraus ergeben sich Verbreitungsbänder, welche mit der Zone 7 an der Felslitoralgrenze beginnen, um gegen die Zone 1 (2 oder 3, welche immer als letzte ausgebildet sein mag) im Brandungsgebiet der am stärksten exponierten Küstenstellen zusammenzurüdken (Abb, 10). Ordnet man aber diesen Verhältnissen die Sedimente zu, die in den jeweils verschiedenen Tiefen den anstehenden Felsen unter sich begraben, also die eigentliche Felslitoral-Grenze bilden, so stellt sich heraus, daß an dieselbe, wiewohl sie sehr einheitlich durch die Hydroiden-Zone 7 zu kennzeichnen war, ganz verschiedene Sedimente grenzen. Auf Geröll und Kies folgen z. B. Grob- und Mittelsande und sandiger Schlamm. Nachdem aber evident wurde, daß sich die Hydroidenzonen (RIEDL 1959, 1964b) ebenso wie die Sedimente (Dietrich \& Kalle 1957, Kuenen 1950, Sverdrup et al. 1954) nach den Expositionsbedingungen anordnen, muß man annehmen, daß es zwei verschiedene Aus schnitte der Exposition sind, die zu so unterschiedlicher Anordnung Anlaß geben. Ich möchte diese Erscheinung mit der Annahme erklären, daß die Hydroidenzonen den Mittelwerten der Teilchengeschwindigkeit folgen, für die Anordnung der Sedimente aber Maxima mittlerer Zeitintervalle verantwortlich sind. Es mag für die Erhaltung eines gröberen Sedimentcharakters genügen, wenn er in Monatsabständen zureichend nachsortiert wird. Mittel- und Maximalwerte zeigten damit in Felslitoralstreifen unterschiedlich geneigte Gefälle. Nach Lage der hydrodynamischen Verhältnisse scheint das auch tatsächlich begründet, denn es ist damit zu rechnen, daß maximal hoher Seegang, wiewohl viel seltener als exponierte Kaps, auch die engeren Buchten treffen kann, welche aber hinsichtlich der mittleren Expositionsverhältnisse gegen jene aber noch stärker zurückfallen.

Die Minima sind in ihrer biologischen Wirkung gleich den Maxima von signifikanten Ausschnittslängen abhängig, jedoch in noch viel einschneidenderem Maße. Hat man erkannt, daß die Existenz eines Sedentariers, etwa nach dem Aspekt der Substratfestigkeit oder dem der Resistenz des Individuums, von einer einzigen, nur Bruchteile von Sekunden dauernden Bewegungsspitze abhängen kann, so lernt man einsehen, daß kurzzeitige Minima überhaupt keine Bedeutung haben. Die Wasserbewegung kann kurzzeitig völlig wegfallen, ohne Einfluß zu nehmen. Ein extremes Exempel bildet der schwingende Wasserkörper, dessen Teilchen in Bodennähe jedesmal beim Wenden der Bewegungsrichtung (beim Kentern des Stromes) einen Augenblick zur Ruhe kommen. Es kommt also nur auf die Dauer der Stagnationsperioden an. Unsere Kenntnisse von Dauer und Wirkung der Minima unter dem Wasserspiegel sind (wie bei den Extremwerten überhaupt) zu gering, um aus diesem Gebiet verläßliche Beispiele zur Hand zu haben. Beobachtungen in Aquarien aber lassen dieselben auch im natürlichen Milieu voraussehen.

Schwämme beispielsweise lassen die Abstufung der nicht mehr erträglichen Dauer von Minimalwerten hinsichtlich der Aspekte Ernährung, Lüftung und Säuberung erkennen. In großen Mengen gefilterten Seewassers vermögen viele Formen wochenlang $\mathrm{zu}$ vegetieren, in geringen, stagnierenden Wassermengen einige Stunden überdauern, wird aber deckender Schlammüberzug nicht entfernt, so können schon nach Stunden irreparable Schäden eintreten. 


\section{DIE KOMPLEXEN WIRKUNGEN}

Neben den Aspekten und den Ausschnitten der Wirkungsweisen, welche in der Regel nur mit jeweils einzelnen Erscheinungen der Wasserbewegung und mit einzelnen Bewegungsweisen verknüptt erscheinen, gibt es biologische Entsprechungen, die mit ganzen Gruppen, ja mit der Gesamtheit der Erscheinungen der Wasserbewegung ursächlich verbunden sein mögen. Man erkennt solche komplexe Beziehungen unter allgemeineren, biologischen Gesichtspunkten. Entsprechend ist ihr Umfang noch kaum abzuschätzen, und ich habe mich darauf zu beschränken, mit einer kleinen Auswahl möglichst typischer Beispiele anzudeuten, was bisher an Typen bewegungsklimatischer Beziehungen deutlich geworden ist.

\section{Auswabl der Wuchsformen}

Untersuchungen an Poriferen (zusammengestell in RIEDL 1964b), Hydroiden (RIEDL 1959) und Anthozoen (ABEL 1959) haben sowohl innerhalb von Arten als auch von Gattungen übereinstimmende Reihen von Wuchsformen und Standort-Expositionsverhältnissen nachweislich gemacht. In der Schwammgattung Axinella folgen mit abnehmender Exposition (Abb. 11) die Arten damicornis, verrucosa, polypoides und ev.

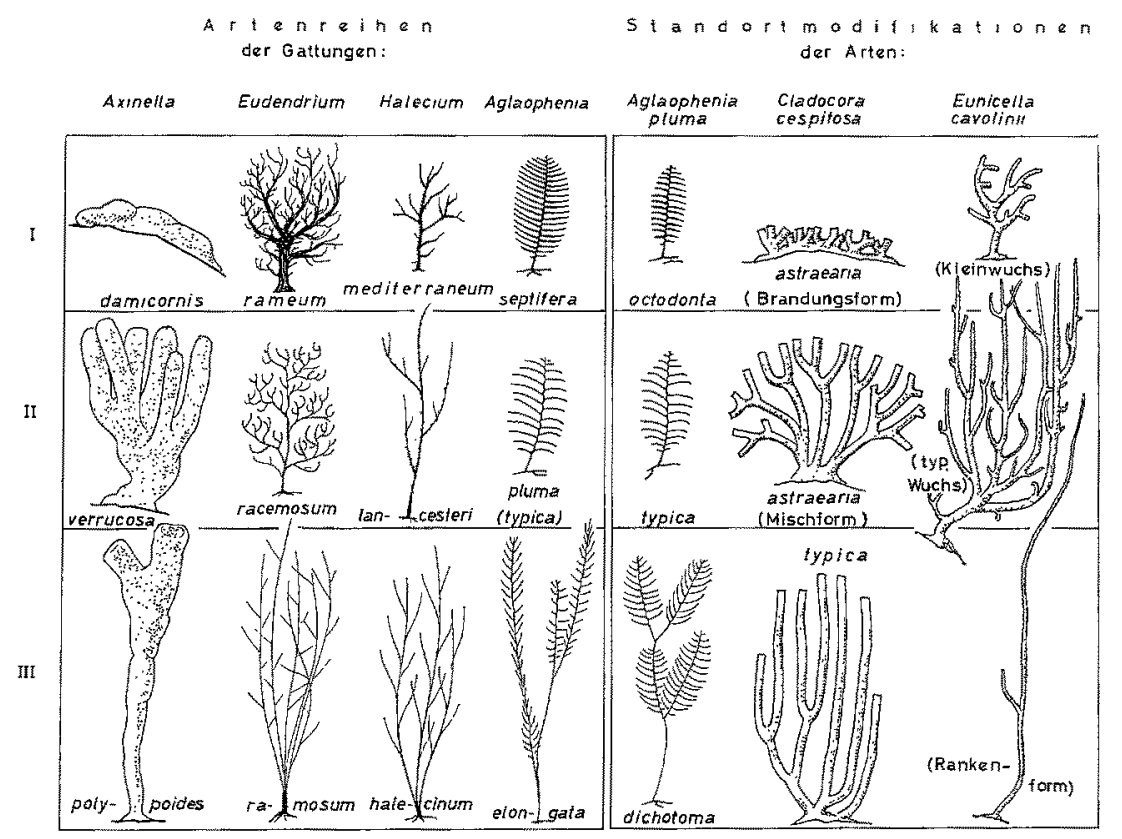

Abb. 11: Die Wirkung der Expositions-Stufen auf die Gestaltsabwandlung innerhalb von Gattungen (Artenreihen) und Ârten (Reihen von Standortmodifikationen). I Formen der Brandungs- und der oberen, stärker exponierten Schwingungszone, If Formen der mittleren und unteren Schwingungszone sowie geschützterer Standorte, III Formen der Strömungszone und sehr geschützter Gebiete. Darstellung der Formen halbschematisch in relativen Größenverhältnissen 
cannabina aufeinander und zeigen eine Gestaltsveränderung vom gedrungen krustenförmigen über den hand- oder baumförmigen bis zum schlank röhrenförmigen Wuchs. Unter den Hydroiden sind es die Genera Eudendrium, Halecium und Aglaophenia, die in gleicher Reihenfolge auf derbe und gedrungene Arten zunehmend schlanke, hohe und locker gebaute Species abfolgen lassen. Unter den Anthozoen deuten sich analoge Verhältnisse bei den Gattungen Cladocora und Eunicella an, die jeweils mit ihren gestreckter wachsenden Arten die geschützten Standorte bevorzugen.

Arten aus diesem Kreise zeigen selbst wiederum Abwandlungen der Wuchsform, die der Exposition der Standortverhältnisse entsprechen. Bei Aglaophenia pluma sind die Formen (wahrscheinlich Standort-Modifikationen) octodonta, typica und dichotoma, bei Cladocora cespitosa sind astraearia-Formen und typica und bei Eunicella cavolinii sind neben kleinwüchsigen und typischen Formen noch lang-rankenförmige Kolonien zu unterscheiden. Auch in diesen Fällen werden die Individuen mit der Geschütztheit der Standorte höher, gestreckter und lockerer. Eine Obersicht über die wahrscheinlich komplexen Ursachen dieser Veränderungen haben wir noch nicht. Wohl liegt auf der Hand, daß der Aspekt der Resistenz maßgeblich beteiligt sein muß. Eine zureichende Erklärung bietet er aber allein nicht. Man wird vielmehr annehmen müssen, daß die Höhe und Lockerheit der Wuchsformen in der Tiefe wahrscheinlich auch die Aussicht auf Verschlammung verringert, die Lüf tung bei Minimalbedingungen begünstigen und selbst den Querschnitt, mit dem Effekt die Durchflu ß menge angehoben zu haben, vergrößert (Abb. 11).

\section{Abwandlung der Filterformen}

Dem Bauplan beispielsweise der großen, polysiphonen Eudendrium-Arten ist es keineswegs vorbestimmt, flächenförmige Wuchsformen $\mathrm{zu}$ entwickeln. Um so auffallender ist die Tatsache, daß selbst das hoch polysiphone Eudendrium rameum an Standorten mit gerichtet pendelndem Wasserkörper, ganz konsequent flächige Stöcke entwickelt. Als Gegenbeispiel (Abb. 12) sind die Arten der Aglaopheniinae anzuführen, deren Stockbau flächigen Wuchs geradezu vorzuschreiben scheint. Diejenigen Formen, die schwingende Wasserkörper oder überhaupt die Hänge des Decliviums besiedeln, Aglaophenia septifera, tubulifera und die forma octodonta der Aglaophenia pluma bleiben auch diesem Prinzip treu. Jene Aglaopheniinen jedoch, die außerhalb des gerichtet bewegten Wassers vorkommen, erreichen auf die verschiedenste Weise die Auflösung des strengen Flächenbaues. Lytocarpia myriopbyllum trägt die Cladien stärker gewinkelt und leicht gekrümmt. Dadurch entsteht aus der Fläche eine Rinne oder ein Halbrohr. Aglaophenia elongata, ebenso auf den tiefen Corallinenböden beheimatet, zeigt den sehr gestreckten und etwas verzweigten Stamm leicht gedreht und etwas gekrümmt. Damit weisen die am weitesten auseinanderliegenden Cladien in verschiedene Richtungen. Und Aglaopbenia pluma forma dichotoma, der man mit einiger Regelmäßigkeit auf Treibgut begegnet, ist oft vielfach verzweigt, wobei der Stamm an jeder Verzweigungsstelle etwas um die Hauptachse gedreht ansetzt. Damit sind die Flächen der einzelnen Cladien-Gruppen wiederum schrittweise gegeneinander verdreht.

Neben den Möglichkeiten der Abwandlung, die selbst in so strengen Bauplänen stecken, zeigen diese Beispiele, daß es nicht nur darauf anzukommen scheint, im ge- 


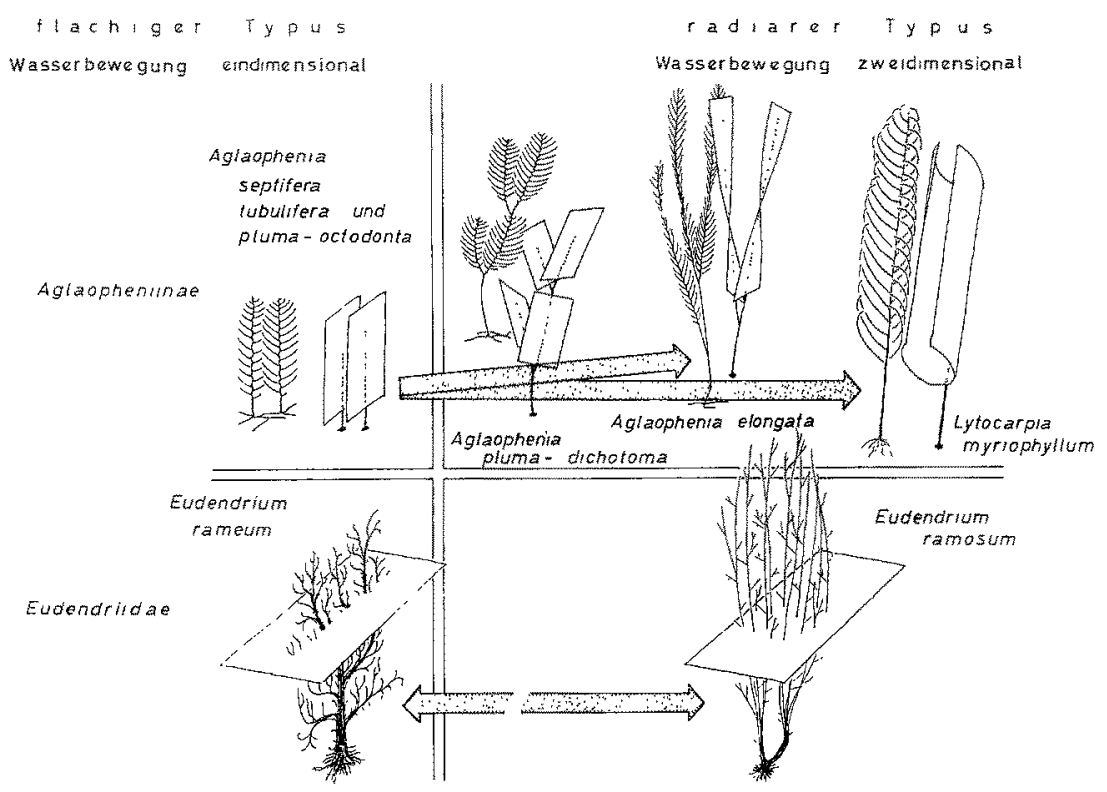

Abb. 12: Die Wirkung der Anzahl der Bewegungs-Dimensionen auf die Abwandlung von Wuchsformen innerhalb nahe verwandter Formen. Die Pfeile deuten die mutmaßliche Lesrichtung der Abwandlung an. Die weißen Flächen symbolisieren bei den Aglaopheniinen das das Schema der Wuchsform, bei den Eudendrien weisen sie auf die Querschnitte hin

richtet bewegten Wasser Flächenformen zu bilden, sondern auch darauf, an Standorten, die aus allen Richtungen beströmt werden, flächige Wuchsformen wiederum aufzulösen. Das Prinzip, den örtlichen Strömungen möglichst häufig mit einem op ti malen Querschnitt gegenüberzustehen, scheint bei diesem Typus der "Cnidenfänger" (einer sehr gestaltenreichen Gruppe "passiver Filtrierer") von recht allgemeiner Bedeutung zu sein.

\section{Wuchsformen und Bestandsdichte}

Ein weiteres Beispiel für die Vielfalt der Beziehungen von Wuchsformenanordnung und Expositionsgröße liefern die Hydroiden, wenn man gleichzeitig die Dichte der Bestände ins Auge faßt. Die Hydroidenbestände der Tyrrhenia-Höhlen beispielsweise zeigen in den sieben Zonen (RIEDL 1959) ein auffallendes Dichtegefälle gegen die schwach exponierten Gebiete. In Hydranthen pro Quadratzentimeter ausgedrückt fallt die Dichte im Mittel von 5000 ubber $1000,300,200$ und 100 auf 40 und 10 in der Zone 7 (Abb. 13). Zudem stellt sich heraus, daß auch die Wuchsformen nicht wahllos über das beschriebene Gefälle verteilt sind. Die nach dem Prinzip des Bauplanes an Hydranthen reichsten und ärmsten Formen (Typus Aglaophenia und Campanularia) stehen an den Enden der Reihen. Zwischen ihnen macht sich (gemessen an der Hydranthenzahl der Stämmchen) ein allgemeines Gefälle von Wuchsformen deutlich. Die ge- 
wisse Unregelmäßigkeit des Wudhs-Gefälles zwischen den Extremen (Abb. 13) hängt damit zusammen, daß die einzelnen Stockformen nicht ohne weiteres verglichen werden können. Betrachtet man sie gruppenweise, so wird das Bild ungleich klarer. So zeigen die in der Reihe aufeinanderfolgenden Plumulariidae (Aglaophenia septifera, Aglaophenia pluma f. octodonta, A. p. f. typica, Plumularia setacea und die Ante-

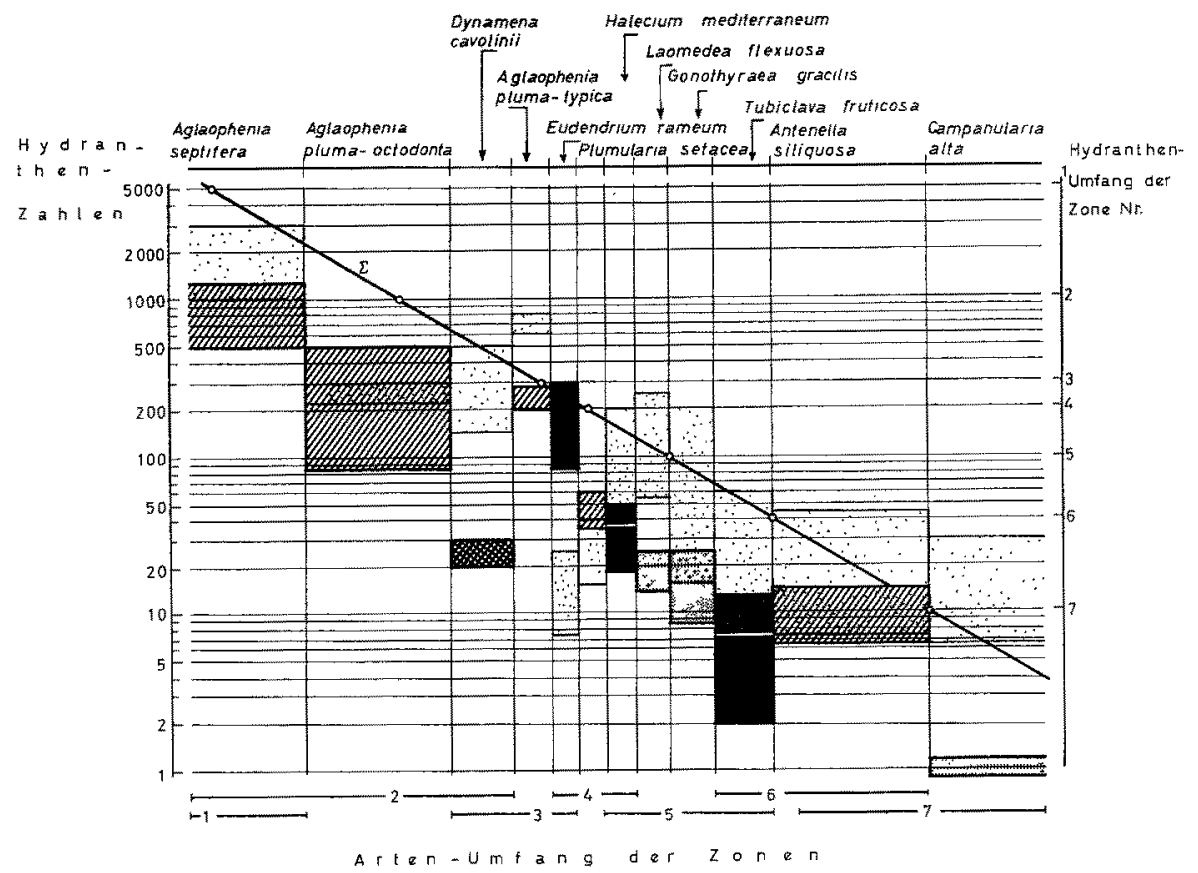

Abb. 13: Die Wirkung der Expositions-Stärke auf die aus Wuchsform und Wuchsenge zusammengesetzte Bestandsdichte, dargestellt am Beispiel der sieben Hydroidenzonen. Angeführt sind die 12 wichtigsten Arten (die Breite der Felder der Arten ist so gewählt, daß die mittleren Hydrantenzahlen pro $\mathrm{cm}^{2}$ je Zone auf einer Geraden liegen). Die punktierten Staffeln geben den Bereich der minimalen bis maximalen Hydrantenzahl der Arten ebenfalls je $\mathrm{cm}^{2}$ an; die dunkel ausgewiesenen. Staffeln (und zwar Plumulariidae gestreift, Sertulariidae kariert, thekenlose Arten schwarz, Plumulariidae grau) repräsentieren die minimalen bis maximalen $\mathrm{Hy}$ dranthenzahlen je Stöckchen. Beachte das regelmäßige Ansteigen jeweils der grauen, schwarzen und gestreiften Staffeln

nella-Arten) ein Gefälle der maximalen Hydranthenzahl von 1200 über 500 und 250 auf 60 und 18. Die Maxima der thekenlosen Formen, also praktisch der Athecata und Halecijdae (Eudendrium rameum, Halecium mediterraneum und Tubiclava fruticosa), sinken von 300 über 50 auf 16. Und die Mittelwerte der Campanulariidae (Laomeda flexwosa, Gonotbyraea gracilis und Campanularia alta, letztere stets unverzweigt) fallen von rund 15 über 8 auf 1 . Zudem stellt man fest, daß Arten mit auffallend großem oder kleinem Wuchs ihre Einpassung in die dem Ort entsprechende Hydranthendichte durch besondere Weite oder Enge der Siedeldichte kompensieren (vgl. Dynamena cavolinii und Campanularia alta gegenüber Eudendrium rameum).

Man erkennt, daß sowohl die einzelnen Wuchstypen, als auch deren Abwandlun- 
gen ihre abgestuften Plätze zugewiesen haben, und man wird annehmen dürfen, daß mit abnehmendem Wasserwechsel auch die Chancen zureichender Ernährung, Lüftung und Säuberung sinken. Diese in Minimalausschnitten wirkenden Aspekte wären geeignet, das geringere Vordringen der hydranthenreichen Wuchsformen gegen die ruhigen Höhlentiefen zu erklären, vorausgesetzt allerdings, daß die hydranthenreichen Bautypen der Hydroiden auch die anspruchsvolleren wären. Um aber einen wenigstens theoretischen Ansatz für die Erklärung der zweiten 'Tatsache zu gewinnen, warum nämlich die hier vorerst als "anspruchslos" etikettierten hydranthenarmen Arten nicht auch in den Gebieten reichlichen Wasserwechsels siedeln, müßte man geeignete Maximal-Aspekte finden. Thre zu geringe Resistenz könnte in Betracht gezogen werden. Wahrscheinlich spielt aber dabei noch Störung und Raumkonkurrenz sowie noch mancher unbekannte Faktor eine Rolle.

\section{Auswabl der Ernährungstypen}

Neben der großen Gruppe der mittels eines eigenen Wasserstromes aktiv sammelnden Filtrierer, in der Hauptsache Porifera, Lamellibranchiata und Ascidiacea, ist eine zweite Gruppe passiver Filtrierer mit vielen Hydroidea und Anthozoa einzurichten; sicher mit allen jenen Formen, welche durch die Ausbildung von Flächen und deren genauer Einstellung in die Bewegungsrichtung des Mediums die Funktion ihres Sammelapparates haben erkennen lassen. Eine $Z$ wischenstellung nehmen die Seepocken ein, die einmal durch den Cirrenschlag zur Gruppe der aktiven Filtrierer zu rechnen sind, ein andermal aber, bei sehr heftiger Wasserbewegung auch eine passive Phase einlegen können, indem sie die Cirrenreuse steif in das vorbeischießende Wasser halten. Formen der Brandungszone, Balanus perforatus sowie Chtbamalus stellatus haben solches Verhalten gezeigt (RIEDL 1964b).

Vergleicht man die Verteilung der beiden Filtrierergruppen und die Abwandlung ihrer Formen über Standorte mit unterschiedlichen Expositionsgraden, vereinfacht entlang von Tiefenreihen, so ergeben sich beträchtliche Unterschiede. Die Bestände der passiven Filtrierer machen mit zunehmender Tiefe einen raschen Formenwandel durch. Verhältnismäßig schmale Verbreitungsbänder folgen in dichter Reihe aufeinander, und jene Arten, die sich über ein etwas größeres Expositionsintervall verbreiten, zeigen eine auffallende Abwandlung der äußeren Form (z. B. Eudenrium, Aglaophenia, Eunicella, Cladocora). Die aktiven Filtrierer dagegen weisen in der Regel ungleich weniger schmale Verbreitungsbänder auf, die Ascidien und Lamellibranchier zudem kaum eine Abwandlung der Gestalt. Nur bei den Poriferen kennt man im Zusammenhang mit veränderten Expositionsverhältnissen ausgeprägtere Wuchsunterschiede; aber auch diese bleiben hinter jenen der passiven Filtrierer weit zurück. So findet sich in den $30 \mathrm{~m}$ tief gelegenen Felslitoral-Schattengebieten eine Vielzahl jener Muschel- und Schwammarten, und noch dazu in ganz unveränderter Gestalt wieder, die bereits dicht unter der Wasserlinie vorkommen. Die endolithischen Arten unter ihnen, die Bohrschwämme und Bohrmuscheln (zum Beispiel Cliona, Litbodomus, Gastrocbaena) machen die Unabhängigkeit aktiver Filtrierer von Expositionsbedingungen besonders klar. Kaum einen passiven Filtrierer aber wird man an beiden Orten zugleich antreffen, und wenn, dann 
nur in so abgewandelter Gestalt, daß man die Individuen vorerst zu verschiedenen Arten stellen wollte, wie das auch tatsächlich oft geschehen ist.

Die Ursache für so ungleiches Standortverwalten wird man in den sehr verschieden engen Umweltbeziehungen der beiden. Typen zu suchen haben. Die aktiven Filtrierer besitzen Innenfilter (oder vermögen sie, wie die Balaniden und viele sedentäre Polychaeten, jedenfalls einzuzichen), die passiven Filtrierer tragen ihren Fangapparat fast immer außen. Und selbst wenn die Polypen kontrahiert oder selbst in Theken zurückgezogen werden können, wird doch der tätige Apparat ganz den Faktoren des Milieus, der Stärke, Richtung und dem Rhythmus der Strömung exponiert. Die Mehrzahl der aktiven Filtrierer hat ein inneres Strömungsmilieu geschaffen, und nur die Außenhaut wird durch Scheuern und Verschütten sowie auf ihre Resistenz geprüft. Die Mehrzahl der passiven Filtrierer dagegen bleibt den primären Milieufaktoren gänzlich ausgesetzt.

\section{ZUSAMMENFASSUNG}

1. Die Wasserbewegung erweist sich als ein Umweltfaktor von erstrangiger Bedeutung. Sie bestimmt weitgehend die Verteilung der Substrate, Anordnung und Gestalt der Organismen, besonders der Sedentarier im Felslitoral. Dennoch ist sie in letzterem noch nicht vermessen worden, denn auf Grund der Vielfalt ihrer Erscheinungen und mutmaßlichen Wirkungsweisen muß vorerst untersucht werden, welche Ursachen und Wirkungen überhaupt verknüpft erscheinen.

2. Die Vereinigung zweier Erfahrungsbereiche macht nun eine erste Zuordnung der hydrodynamischen Erscheinungen noch vor deren Vermessung im Biotop möglich: Ozeanographie, Geologie, Geomorphologie und Wasserbau bieten zusammen ein so großes, einschlägiges Material, daß schon eine Vielzahl von Bewregungserscheinungen im Litoral vergleichend erkannt werden kann. Die wissenschaftliche Taucherei im Litoral ließ durch die gewonnene Anschauung eine Fülle von Ursachen und Wirkungen verknüpfen.

3. Die bislang erkannten etwa 27 unterschiedlichen Verknüpfungsweisen werden nach 5 Gesichtspunkten analysiert und mit je einem Beispiel belegt. Der künftigen metrischen Bearbeitung sollen damit die Ansatzpunkte geliefert werden.

4. Grunderscheinungen: Die Staudruckwirkung läß sich an Substrat- und Organismenfestigkeit, die der Durchflußmenge an Ernährung und Belüftung ablesen, jene der Wasserversetzung, z. B. an der Medusenverdriftung. Die Teilchengeschwindigkeit bildet die allgemeinste Meßeinheit.

5. Bewregungsweisen: Der streichende Wasserkörper trennt in seinen zwei Formen flächige und radiäre Wuchstypen, der schwingende verändert die Orientierung der flächigen Formen, der zerreißende führt zur Auslese von z. B. gepanzerten Schildformen.

6. Expositionsgefälle: Die Küstenexposition ist etwa nach den Halophyten-, Lithophyten- und Hydroidenzonen zu bemessen, das Gefälle bei zunehmender Tiefe nach der Orientierung der Hydroiden und Anthozoen in den kritischen Tiefen. Die Wirkung der Substratgestalt läßt sich der Hydroiden-Anordnung, die der Substratgliederung jener der Mikrofauna entnehmen. 
7. Einfache Wirkungsweisen: Jeweils nach den Aspekten und den Ausschnitten (welche die Wirkungen bestimmen) getrennt dargestellt:

a) Die Aspekte: Die Bedeutung der Substratfestigkeit wird durch die Aufwuchsverhältnisse in Blockfeldern, die der Störung durch die Hydroiden-Auswahl in Tangbeständen, die der Resistenz am Beispiel der Brandungs-Selektion erörtert; jene der Verschüttung, der Säuberung und des Scheuerns mit Vorgängen am Felslitoral-Rand, in Höhlen und Geschiebeböden belegt. Der Aspekt Ernährung wird an Hand des Wasserdurchzuges der höhlenbewohnenden Filtrierer, der der Lüftung am Beispiel der Abgabe von Fortpflanzungsprodukten geschildert. Befruchtung und Verbreitung hängen wiederum mit der Wasserversetzung zusammen.

b) Die wirkenden Ausschnitte: Die Kennzeichen der Maxima werden aus dem Verhältnis Lithophyten- zu Halophytenzone, die der Mittelwerte aus dem der Hydroiden- und Sedimentzonen an den Felslitoralgrenzen abgeleitet, jene der Minima aus Mortalitätsverhältnissen unter künstlichen Bedingungen.

8. Komplexe Wirkungen: Expositionsbedingte Wuchsformen-Auswahl wird durch Reihen von Arten und Standortmodifikationen belegt, die Abwandlung der Filterformen an Hand der flächigen Hydroiden geschildert. Wuchsform und Bestandsdichte in ihrer korrelierten Abhängigkeit lassen die Hydroidenzonen der Höhlen erkennen. Die unterschiedliche Expositionsabhängigkeit der Ernährungstypen zeigt die Gegenüberstellung der aktiven und passiven Filtrierer.

\section{ZITIERTE LITERATUR}

ABEL, E., 1959. Zur Kenntnis der marinen Höhlenfauna unter besonderer Berücksichtigung der Anthozoa. Ergebnisse der Osterr. Tyrrhenia-Expedition 1952, Teil V. Pubbl. Sta. zool. Napoli, Suppl. 30, 1-94.

Cvıjıć, J., 1924. Morphologie terrestre (serbisch). Vol. 1. Drschawna Schtamparija, Belgrad, $588 \mathrm{pp}$.

Defant, A., 1929. Dynamische Ozeanographie. (Einführung in die Geophysik, T. 3.) Springer, Berlin, $222 \mathrm{pp}$.

Dietrich, G. \& Kalde, K., 1957. Allgemeine Meereskunde. Eine Einführung in die Ozeanographie. Borntraeger, Berlin-Nikolassee, $492 \mathrm{pp}$.

Domzig, H., 1955. Wellendruck und druckerzeugender Seegang. Mitt. hann. VersAnst. Grundban 8, 1-79.

GUilcher, A., 1954. Morphologie littorale et sous-marine. Presses Univ., Paris, 216 pp.

Iribarren, R., 1953. Beim Brechen von Wellen unvermutet auftretende heftige Drucke. Mitt. bann. VersAnst. Grundban 3, 4-9.

JØRGENSEN, G. B., 1955. Quantitative aspects of filter feeding in invertebrates. Biol. Rev. 30, $391-454$.

Kuenen, P., 1950. Marine geology. John Wiley \& Sons, New York, 568 pp.

Lorenz, J. R., 1863. Physikalische Verhältnisse und Vertheilung der Organismen im Quarnerischen Golfe. Geroldson, Wien, $379 \mathrm{pp}$.

MAGNus, D., 1963a. Über das "Abweiden" der Flutwasseroberfläche durch den Schlangenstern Ophiocoma scolopendrina (Lamarck). Zool. Anz. (Suppl.) 26, 471-481.

- 1963b. Der Federstern Heterometra savignyi im Roten Meer. Natur Mus., Frankf. 93, 355-368.

RreDL, R., 1956. Automatische Photographie von Meeresböden für ökologisch-faunistische Zwecke. Öst. zool. Z. 6, 532-541. 
RIEDL, R., 1959. Die Hydroiden des Golfes von Neapel und ihr Anteil an der Fauna unterseeischer Höhlen. Ergebnisse der Osterr. Tyrrhenia-Expedition 1952, Teil XVI. Pubbl. Sta. zool. Napoli, Suppl. 30, 589-755.

- 1963. Probleme und Methoden der Erforschung des litoralen Benthos. Zool. Anz. (Suppl.) 26, 505-567.

- 1964a. 100 Jahre Litoralgliederung seit JOSEF LORENZ, neue und vergessene Gesichtspunkte. Int. Rev. ges. Hydrobiol. 49, 281-305.

- 1964b. Biologie der Meereshöhlen. Deskriptive Beiträge zur Monographie eines Lebensraumes. Gustav Fischer, Stuttgart (etwa 400 pp., in Vorbereitung).

RÜTZLER, K., 1962. Systematik und Ókologie der Poriferen aus Litoral-Schattengebieten der Nordadria. Dissertation Wien, $138 \mathrm{pp}$.

Shepard, F., 1948. Submarine geology. Harper Br., New York, 348 pp.

Sverdrup, H., Johnson, M. \& Fleming, R, 1954. The Oceans, their physics, chemistry and general biology. Prentice-Hall, New York (5. Aufl.), 1060 pp.

Thorade, H., 1931. Probleme der Wasserwellen. In: Probleme der kosmischen Physik. Hrsg. von C. Jensen u. A. Schwassmann. Bde 13., 14. Akad. Verl.-Ges., Leipzig, 219 pp.

VollbrechT, K., 1954. Beiträge zum Problem brandender Wellen. Acta bydrophys. 2, 10-30.

WERNER, B., 1951. Uber die Bedeutung der Wasserstromerzeugung und Wasserstromfiltration für die Nahrungsaufnahme der ortsgebundenen Meeresschnecke Crepidula fornicata L. (Gastropoda, Prosobranchia). Zool. Anz. 146, 97-113.

- 1953. Ausbildungsstufen der Filtermechanismen bei filtrierenden Prosobranchiern. Zool. Anz. (Suppl.) 17, 529-546. 